\title{
EXISTENCE AND STABILITY OF STANDING PULSES IN NEURAL NETWORKS: I. EXISTENCE
}

\author{
YIXIN GUO* AND CARSON C. $\mathrm{CHOW}^{\dagger}$
}

\begin{abstract}
We consider the existence of standing pulse solutions of a neural network integrodifferential equation. These pulses are bistable with the zero state and may be an analogue for short term memory in the brain. The network consists of a single-layer of neurons synaptically connected by lateral inhibition. Our work extends the classic Amari result by considering a non-saturating gain function. We consider a specific connectivity function where the existence conditions for singlepulses can be reduced to the solution of an algebraic system. In addition to the two localized pulse solutions found by Amari, we find that three or more pulses can coexist. We also show the existence of nonconvex "dimpled" pulses and double pulses. We map out the pulse shapes and maximum firing rates for different connection weights and gain functions.
\end{abstract}

Key words. integro-differential equations, integral equations, standing pulses, neural networks, existence

AMS subject classifications. 34A36, 37N25, 45G10, 92B20

1. Introduction. The temporary storage of information in the brain for short periods of time is called working memory [6]. It is known that the firing activity of certain neurons in the cortex are correlated with working memory states but it is not known what neural mechanisms are responsible for maintaining the persistent neural activity 25, 28, 70]. Experiments find that a specific set of neurons become activated by a memory cue. They fire at a rate above their background levels while the memory is being held and then return to baseline levels after the memory is extinguished. When the neurons are active their firing rates are low compared to their maximal possible rates. Cortical neurons are generally not intrinsically bistable and do not fire unless given an input that is above a threshold [14, 47, 66]. It has been suggested that recurrent excitatory inputs in a network could be responsible for maintaining neural activity observed during memories [25, 28, 31, 47, 66, 69, 70, 72, 73, 34, 44. The persistent activity is bistable with the background state. To match experimental data, a memory network must have the ability to maintain persistent activity in a selected subset of the neurons while keeping the firing rates low compared to their possible maximum.

Mathematically, this question has been probed by examining the existence and stability of localized persistent stationary solutions of neural network equations 3 15. 20, 21, 31, 57, 63, 72, 73. These localized states have been dubbed 'bump attractors' 43, 41, 44, 34, 70, 72. In a one dimensional network they have also been called standing pulses [20, 57]. While these simple networks do not capture all of the biophysical features of cortical circuits they do capture the qualitative behavior of working memory.

The coarse-grained averaged activity of a neural network can be described by $[3$ 20, 31, 72, 73 .

$$
\tau \frac{\partial u(x, t)}{\partial t}=-u(x, t)+\int_{\Omega} w(x-y) f[u(y, t)] d y
$$

\footnotetext{
*Department of Mathematics, The Ohio State University, Columbus, OH 43210 (yigst@math.ohio-state.edu).

${ }^{\dagger}$ Department of Mathematics, University of Pittsburgh, Pittsburgh, PA 15260 (ccchow@pitt.edu).
} 
where $u(x, t)$ is the synaptic input to neurons located at position $x \in(-\infty, \infty)$ at time $t \geq 0$, and it represents the level of excitation or amount of input to a neural element. The connection function $w(x)$ determines the connections between neurons. The nonnegative and monotonically non-decreasing gain function $f[u]$, denotes the firing rate at $x$ at time $t$. We can set the synaptic decay time $\tau$ to unity without loss of generality.

In his classic work, Amari 3] considered (1.1) with a 'Mexican Hat' connection function (i.e. excitation locally and inhibition distally). While this is not biologically realistic for a single layer of neurons, it has been argued that networks of combined excitatory and inhibitory neurons with biophysically plausible connections can effectively mimic a Mexican Hat under certain conditions [20, 37, 57]. Amari also made the assumption that $f[u]$ is the Heaviside function. This approximation made (1.1) analytically tractable and he was able to find a host of solutions one of them being localized stable pulses that are bistable with zero activity. Kishimoto and Amari 39] later showed these solutions also existed for a smooth sigmoidal gain function that saturated quickly.

Later work considered two populations [56, 57, various connectivity functions 15] 43, 63, and two dimensions [36, 44]. However, all used either the Heaviside gain function or a saturating sigmoidal gain function implying that neurons start to fire when their inputs exceed threshold and saturate to their maximum rate quickly. However, in the brain persistently active neurons fire at rates far below their saturated maximum [12, 13, 24, 69, 70]. How a network can maintain persistent activity at low firing rates is not fully understood [10, 13, 31, 43, 45, 46, 61, 69, 70].

The problem of persistent activity at low firing rates cannot be addressed with a quickly saturating gain function. To circumvent this limitation, we use a nonsaturating piecewise-linear gain function with a jump i.e. $\beta=0$ (see Fig. 2.2) having the form

$$
g[u]= \begin{cases}\alpha\left(u-u_{T}\right)+\beta & u>u_{T} \\ 0 & u \leq u_{T}\end{cases}
$$

When the gain $\alpha$ is zero, (1.2) becomes the Heaviside function scaled by $\beta$. We note that others have considered piecewise linear gain functions but without the jump [7] [35. 67. In these cases, persistent activity is not possible unless the threshold is set to zero and the gain to unity where a multi-stable "line attractor" is possible 67.

In this paper we show the existence of isolated convex standing pulse solutions (single-pulses) of (1.1). We consider a single one dimensional layer of neurons. Although, this configuration is a major simplification, it has been shown that such networks exhibit features present in more realistic architectures. We investigate how the pulse solutions change when parameters of the gain function and the connection function change. We demonstrate the coexistence of two single-pulse solutions as seen by Amari [3], and give conditions where more than two pulse solutions can coexist. We also show the existence of nonconvex "dimple-pulse" solutions and double-pulse solutions. We derive the stability criteria for stable pulses in an accompanying paper 33.

2. Neural network equations. We study a neural network (1.1) with lateralinhibition or Mexican Hat type connection function $w(x)$ for which excitatory connections dominate for proximal neurons and inhibitory connections dominate for distal neurons. In general, $w(x)$ satisfies the following six properties.

1. $w(x)$ is symmetric, i.e. $w(-x)=w(x)$, 
2. $w(x)>0$ on an interval $\left(-x_{0}, x_{0}\right)$, and $w\left(-x_{0}\right)=w\left(x_{0}\right)=0$,

3. $w(x)$ is decreasing on $\left(0, x_{0}\right]$;

4. $w(x)<0$ on $\left(-\infty,-x_{0}\right) \cup\left(x_{0}, \infty\right)$;

5. $w(x)$ is continuous on $\Re$, and $w(x)$ is integrable on $\Re$;

6. $w(x)$ has a unique minimum $x_{m}$ on $\Re^{+}$such that $x_{m}>x_{0}$, and $w(x)$ is strictly increasing on $\left(x_{m}, \infty\right)$.

For concreteness, we consider the connection function given by

$$
w(x)=A e^{-a|x|}-e^{-|x|}
$$

where $a>1$, and $A>1$ guarantee that $w(x)$ obeys properties 1 - 6. An example of (2.1) is shown in Fig. 2.1. This connection function is of the lateral inhibition or Mexican Hat class. Perhaps, given the cusp at zero it should be called a "Wizard Hat" function.

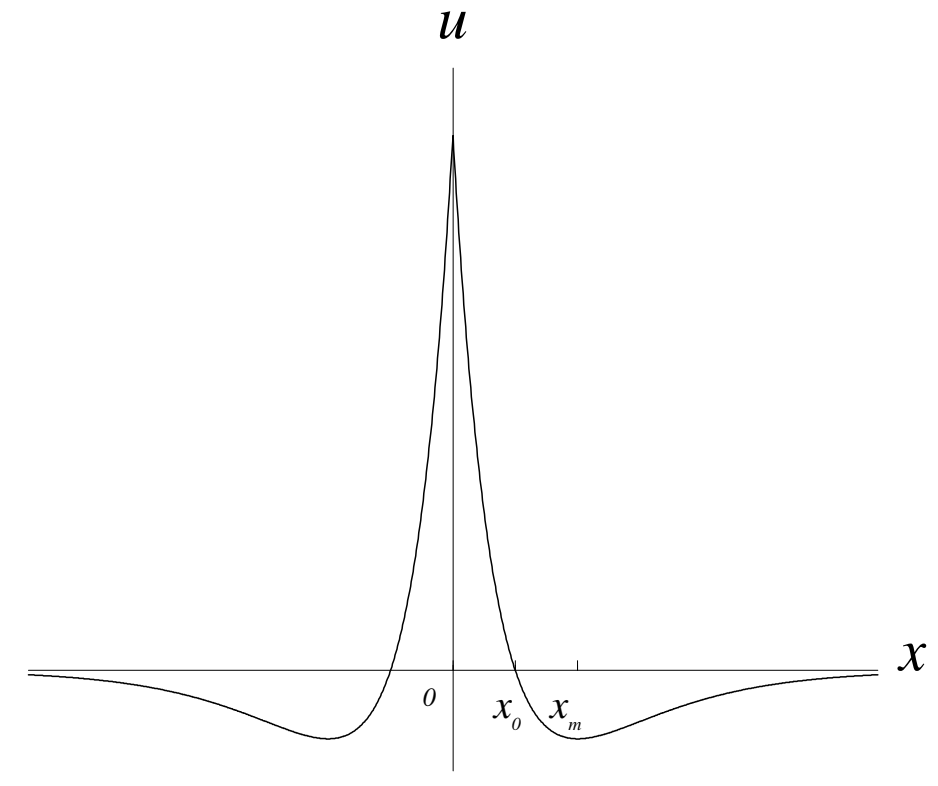

FIG. 2.1. Connection function with $A=2.8, B=1, a=2.6, b=1$.

For connection function (2.1), $x_{0}=\frac{\ln A}{a-1}$ and $x_{m}=\frac{\ln a A}{a-1}$. The area of $w(x)$ above and below the $x$-axis represents the net excitation and inhibition in the network respectively. The total area of (2.1) is $2\left(\frac{A}{a}-1\right)$. The amount of excitation and inhibition depends on the ratio of $A$ to $a$. If $A>a$, i.e. $2\left(\frac{A}{a}-1\right)>0$, excitation dominates in the network and if $2\left(\frac{A}{a}-1\right)<0$, inhibition dominates. In the balanced case, $A=a$, i.e. $2\left(\frac{A}{a}-1\right)=0$.

The gain function (1.2) can be written as

$$
f[u]=\left[\alpha\left(u-u_{T}\right)+\beta\right] \Theta\left(u-u_{T}\right)
$$

where $\Theta\left(u-u_{T}\right)$ is the Heaviside function such that

$$
\Theta\left(u-u_{T}\right)=\left\{\begin{array}{ll}
1 & \text { if } u>u_{T} \\
0 & \text { otherwise }
\end{array} .\right.
$$




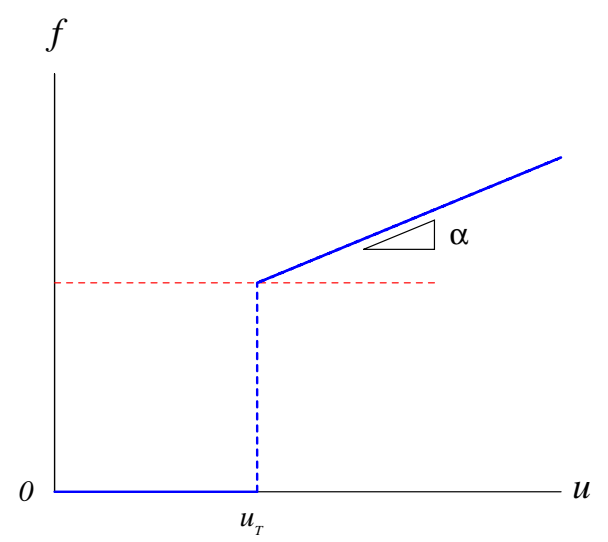

FIG. 2.2. Piecewise-linear gain function.

The gain function (2.2) does not saturate with a positive slope $\alpha$. Without loss of generality, we set $\beta=1$. The gain function (2.2) turns into the Heaviside function when $\alpha=0$ ( See Fig. 2.2).

A stationary solution of (1.1) satisfies the equilibrium equation

$$
u(x)=\int_{-\infty}^{\infty} w(x-y) f[u(y)] d y .
$$

An example of a working memory state can be seen by considering constant solutions of (2.4). For $u(x)=u^{0}$, the integral equation becomes

$$
u^{0}=f\left[u^{0}\right] \int_{-\infty}^{\infty} w(y) d y
$$

Using (2.1), the constant solution satisfies

$$
u^{0}=w^{0} f\left[u^{0}\right] .
$$

where $w^{0}=2(A / a-1)$. From (2.6), we immediately see that $u^{0}=0$ is a solution. In fact, zero is a solution of (2.4) for any positive threshold $u_{T}$ and any values of parameters $a, A$, and $\alpha$.

Inserting gain function (2.2) into (2.6) gives

$$
u^{0}=w^{0}\left(\alpha\left(u^{0}-u_{T}\right)+1\right)
$$

The existence of constant solutions can be deduced graphically (see Fig. 2.3). Nontrivial constant solutions $\left(u^{0}>0\right)$ require $w^{0}>0$ which means that $A / a>1$. Thus only for net excitatory connections are nontrivial constant solutions possible. A simple stability calculation shows that $\alpha<1$ is necessary for stability. Condition (2.7) shows that for $u_{T}<0$ and $\alpha<1$, there is a single stable solution. If $u_{T}>0$ and $\alpha>0$ there can be three solutions (see Fig. 2.3). Two of the solutions, $u^{0}=0$ and $u^{0}>u_{T}$ are stable. The third solution at $u^{0}=u_{T}$ is unstable. For this parameter set, the network exhibits working memory-like behavior. The network is stable in the background state $u^{0}=0$. A transient input from a memory cue can switch the network into the stable $u^{0}>u_{T}$ state which represents the memory. This is a state of persistent activity that is sustained by positive feedback. The state can be switched off to zero by another transient input when it is no longer needed. The next section will examine spatially localized pulses that have the same memory property. 


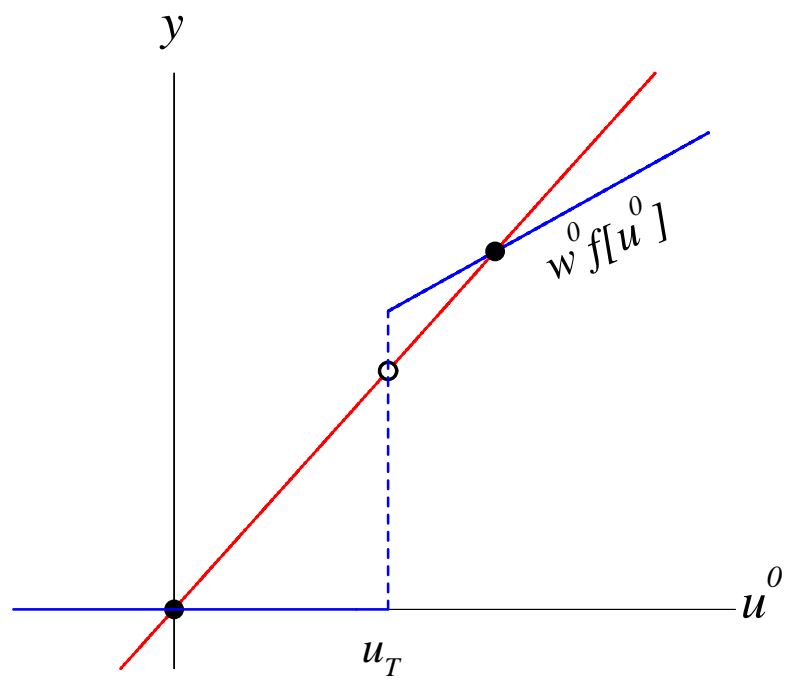

FIG. 2.3. Bistability of constant solutions. The solid circles are the two stable constant solutions and the open circle is an unstable solution. $w^{0}$ is the integral of $w(x)$ on its domain.

3. Single-pulse solutions. We prove the existence and determine the properties of localized stationary persistent states which we call single-pulses. We consider single-pulse solutions of (2.4) that satisfy the following:

Definition 3.1. Single-pulse solution:

$$
u(x) \begin{cases}>u_{T} & \text { if } x \in\left(-x_{T}, x_{T}\right), x_{T}>0 \\ =u_{T} & \text { if } x=-x_{T}, x=x_{T} \\ <u_{T} & \text { otherwise }\end{cases}
$$

such that $\left(u, u^{\prime}, u^{\prime \prime}, u^{\prime \prime \prime}\right) \rightarrow(0,0,0,0)$ exponentially fast as $x \rightarrow \pm \infty$ and $u, u^{\prime} \in L^{1}(\Re)$. $u$ and $u^{\prime}$ are bounded and continuous on $\Re$. $u^{\prime \prime}, u^{\prime \prime \prime}$, and $u^{\prime \prime \prime \prime}$ are continuous everywhere except at $x= \pm x_{T}$ and bounded everywhere on $\Re$. $u(x)$ is symmetric with $u^{\prime \prime}(0)<0 ; u(0)$ is the maximum between $-x_{T}$ and $x_{T}$ ( Fig. [3.1])

We note that there also exist pulses where $u^{\prime \prime}(0)>0$, which implies $u(0)$ is no longer the maximum of the pulse. We call this solution a dimple-pulse. The theorem below gives a range for which there is no single-pulse solution.

Theorem 3.2. For fixed $a, A$ and $\beta=1$, there is no single-pulse solution if both $\alpha<\frac{a}{2 A}$ and $u_{T}>\frac{2 A}{a}$ are true.

Proof. Substituting the exponential connection function (2.1) and gain function (2.2) into the integral equation (2.4) gives

$$
u(x)=\int_{\infty}^{\infty}\left(A e^{-a|x-y|}-e^{-|x-y|}\right)\left[\alpha\left(u(y)-u_{T}\right)+1\right] \Theta\left(u-u_{T}\right) d y .
$$

Suppose there is a single-pulse solution as defined above when both $\alpha<\frac{a}{2 A}$ and $u_{T}>\frac{2 A}{a}$ are satisfied. For a single pulse to exist

$$
u(0)=\int_{-\infty}^{\infty}\left(A e^{-a|y|}-e^{-|y|}\right)\left[\alpha\left(u(y)-u_{T}\right)+1\right] \Theta\left(u-u_{T}\right) d y
$$




$$
\begin{aligned}
& =\int_{-x_{T}}^{x_{T}}\left(A e^{-a|y|}-e^{-|y|}\right)\left[\alpha\left(u(y)-u_{T}\right)+1\right] d y \\
& \leq \int_{-x_{T}}^{x_{T}} A e^{-a|y|}\left[\alpha\left(u(y)-u_{T}\right)+1\right] d y
\end{aligned}
$$

where $u(x) \geq u_{T}$ is continuous on $I:=\left[-x_{T}, x_{T}\right] . A e^{-a|y|}$ is integrable on $I$ and $A e^{-a|y|} \geq 0$ for all $x \in I$. By the Mean Value Theorem for Integrals, $\exists c^{0} \in I$ s.t.

$$
\begin{aligned}
u(0) & \leq\left(\alpha u\left(c^{0}\right)-\alpha u_{T}+1\right) \int_{-x_{T}}^{x_{T}} A e^{-a|y|} d y \\
& \leq \alpha P u\left(c^{0}\right)+\left(1-\alpha u_{T}\right) P
\end{aligned}
$$

where $P=\int_{-\infty}^{\infty} A e^{-a|y|} d y=\frac{2 A}{a}$. If $\alpha P<1$ and $\left(1-\alpha u_{T}\right) \leq 0$ are both true, then $u(0)<u\left(c^{0}\right), c \in I$. However, this cannot be true because $u(0)$ is the maximum of $u(x)$ on $\Re$. From $\alpha P<1$, we get $\alpha<\frac{a}{2 A}$. From $\left(1-\alpha u_{T}\right) \leq 0, u_{T} \geq \frac{1}{\alpha}>\frac{2 A}{a}$. Therefore, there is no single-pulse when both $\alpha<\frac{a}{2 A}$ and $u_{T}>\frac{2 A}{a}$ are both true. In other words if the gain is too low or the threshold too high, there cannot be a single-pulse.

3.1. Strategy to construct a single-pulse solution. The general approach to studying integral equation (2.4) is to derive an associated differential equation whose solutions are also solutions of the integral equation. We derive the differential equation by using the Fourier transform

$$
F[g(x)]=\int_{-\infty}^{\infty} g(x) e^{i s x} d x
$$

where $g \in L^{1}(\Re)$ and $s \in \Re$, with the inverse Fourier transform

$$
g(x)=\frac{1}{2 \pi} \int_{-\infty}^{\infty} F[g(x)] e^{-i s x} d s .
$$

For our conditions on $u(x)$ and $w(x)$, an application of the Fourier transform to (2.4) is well-defined and turns the convolution into a point-wise product

$$
F[u]=F[w] F[f[u]] .
$$

Computing $F[w]$ in (3.3) gives

$$
F[u]=\frac{\left(2 a A+2 a s^{2} A-2 a^{2}-2 s^{2}\right)}{\left(a^{2}+a^{2} s^{2}+s^{2}+s^{4}\right)} F[f] .
$$

Multiplying both sides of (3.4) by the denominator of the right hand side and using the linear property of the Fourier Transform with the identities $F\left[u^{\prime \prime}\right]=-s^{2} F[u]$ and $F\left[u^{\prime \prime \prime \prime}\right]=s^{4} F[u]$ gives

$$
F\left[u^{\prime \prime \prime \prime}-\left(a^{2}+1\right) u^{\prime \prime}+a^{2} u\right]=F\left[2\left(a A-a^{2}\right) f\right]+2(a A-1) F\left[s^{2} f\right] .
$$

By the definitions of $u(x)$ and $f[u]$,

$$
F\left[u^{\prime \prime \prime \prime}-\left(a^{2}+1\right) u^{\prime \prime}+a^{2} u\right]
$$


and

$$
F\left[2\left(a A-a^{2}\right) f\right]
$$

are in $L^{1}(\Re)$.

Integrating $F\left[s^{2} f\right]$ by parts yields

$$
\begin{aligned}
F & {\left[s^{2} f\right] } \\
= & \int_{-\infty}^{\infty} s^{2} e^{i s x} f[u(x)] d x \\
= & \int_{-x_{T}}^{x_{T}} s^{2} e^{i s x} f[u(x)] d x \\
= & f\left[u\left(x_{T}\right)\right]\left(-i s e^{i s x_{T}}+i s e^{-i s x_{T}}\right)+f^{\prime}\left[u\left(x_{T}^{-}\right)\right] u^{\prime}\left(x_{T}\right)\left(e^{i s x_{T}}+e^{-i s x_{T}}\right) \\
& -\int_{-x_{T}}^{x_{T}} e^{i s x} \frac{d^{2} f[u(x)]}{d x^{2}} d x .
\end{aligned}
$$

Note that $f[u(x)]=0$ outside of $\left(-x_{T}, x_{T}\right)$ and $F\left[s^{2} f\right] \in L^{1}(\Re)$.

Applying the inverse Fourier transform to 3.5 gives a fourth order ordinary differential equation

$$
\begin{aligned}
& u^{\prime \prime \prime \prime}-\left(a^{2}+1\right) u^{\prime \prime}+a^{2} u=2\left(a A-a^{2}\right) f[u(x)]+ \\
& 2(a A-1)\left\{f\left[u\left(x_{T}\right)\right] \Delta^{\prime}(x)+f^{\prime}\left[u\left(x_{T}^{-}\right)\right] u^{\prime}\left(x_{T}\right) \Delta(x)-\frac{d^{2} f[u(x)]}{d x^{2}}\right\}
\end{aligned}
$$

where

$$
\Delta^{\prime}(x)=\delta^{\prime}\left(x-x_{T}\right)+\delta^{\prime}\left(x+x_{T}\right)
$$

and

$$
\Delta(x)=\delta\left(x-x_{T}\right)+\delta\left(x+x_{T}\right) .
$$

Here $\delta(x)$ and $\delta^{\prime}(x)$ are defined as [23]

$$
\delta(x)=\int_{-\infty}^{\infty} e^{i s x} d x, \quad \delta^{\prime}(x)=i s \int_{-\infty}^{\infty} e^{i s x} d x .
$$

If $u(x)$ is a solution of (3.6) where (3.3)-(3.5) hold, then $u(x)$ is also a solution of (2.4).

We construct a single-pulse solution as in Fig. 3.1 by decomposing ODE (3.6) into two linear differential equations:

$$
\begin{aligned}
& (3.7) u^{\prime \prime \prime \prime}-\left(a^{2}+1\right) u^{\prime \prime}+a^{2} u=2 a(A-a) f(u)-2(a A-1) \frac{d^{2} f[u]}{d x^{2}} \text {, if } u>u_{T} \text { (region I) } \\
& \text { (3.8) } u^{\prime \prime \prime \prime}-\left(a^{2}+1\right) u^{\prime \prime}+a^{2} u=0, \quad \text { if } u<u_{T} \text { (region II and III) }
\end{aligned}
$$

We label the solution of (3.6) on regions I, II and III, $u_{\mathrm{I}}(x)$ by $u_{\mathrm{II}}(x)$ and $u_{\mathrm{III}}(x)$ respectively. The solutions $u_{\mathrm{I}}(x), u_{\mathrm{II}}(x)$ and $u_{\mathrm{III}}(x)$ must be connected together at $-x_{T}$ and $x_{T}$ to get a continuous and smooth $u(x)$ on $\Re . u_{\mathrm{I}}(x)$ and $u_{\mathrm{II}}(x)$ are connected 


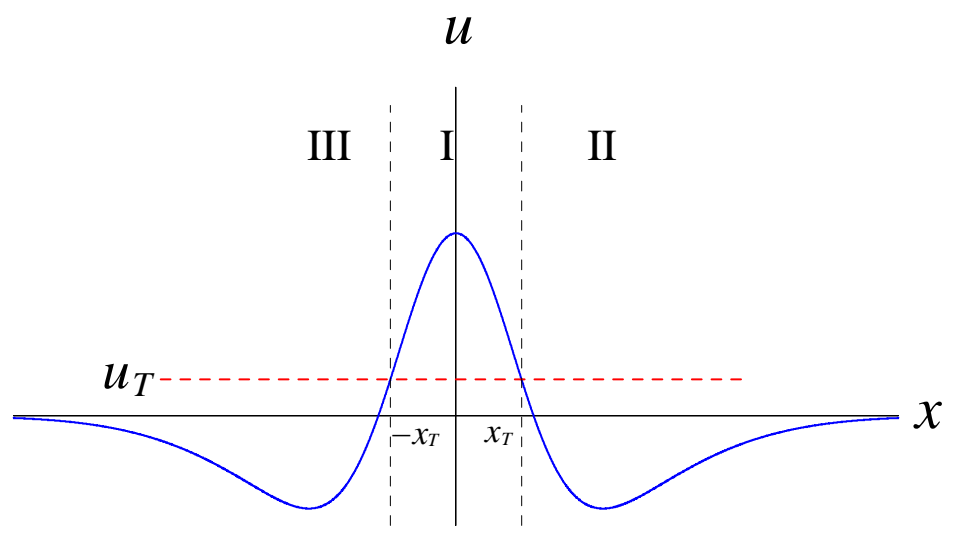

FIG. 3.1. Single-pulse solution.

at $x_{T}$ with five matching conditions:

$$
\begin{aligned}
u_{\mathrm{I}}\left(x_{T}\right) & =u_{T} \\
u_{\mathrm{II}}\left(x_{T}\right) & =u_{T} \\
u_{\mathrm{I}}^{\prime}\left(x_{T}\right) & =u_{\mathrm{II}}^{\prime}\left(x_{T}\right) \\
u_{\mathrm{I}}^{\prime \prime}\left(x_{T}\right) & =u_{\mathrm{II}}^{\prime \prime}\left(x_{T}\right)-2(a A-1) f\left(u\left(x_{T}\right)\right) \\
u_{\mathrm{I}}^{\prime \prime \prime}\left(x_{T}\right) & =u_{\mathrm{II}}^{\prime \prime \prime}\left(x_{T}\right)-2(a A-1) f^{\prime}\left(u\left(x_{T}\right)\right) u^{\prime}\left(x_{T}\right)
\end{aligned}
$$

Conditions (3.9)-(3.11) are given by the continuity of $u(x)$ and $u^{\prime}(x)$. (3.13) is obtained by integrating (3.6) over a small neighborhood of $x_{T}$. (3.12) is obtained by integrating (3.6) twice, first with respect to $x$, then over a small neighborhood of $x_{T} . u^{\prime \prime}(x)$ and $u^{\prime \prime \prime}(x)$ are discontinuous at $x_{T}$, i.e. there are jumps in $u^{\prime \prime}\left(x_{T}\right)$ and $u^{\prime \prime \prime}\left(x_{T}\right)$. Since $u(x)$ is symmetric, similar matching conditions apply to $u_{\mathrm{I}}(x)$ and $u_{\mathrm{III}}(x)$ at $-x_{T}$.

In region II, the solution for a single pulse that satisfies the boundary conditions is

$$
u_{\mathrm{II}}(x)=E e^{-a x}+F e^{-x} \quad E, F \in \Re .
$$

By symmetry, the solution in region III is

$$
u_{\mathrm{III}}(x)=E e^{a x}+F e^{x}, \quad E, F \in \Re .
$$

In region I, substituting $f[u(x)]=\alpha\left(u-u_{T}\right)+1$ and $\frac{d^{2} f[u(x)]}{d x^{2}}=\alpha u^{\prime \prime}(x)$ into (3.7) gives

$$
u^{\prime \prime \prime \prime}-\left(a^{2}+1-2 \alpha(a A-1)\right) u^{\prime \prime}+\left(a^{2}-2 a \alpha(A-a)\right) u=2 a(A-a)\left(1-\alpha u_{T}\right)
$$

The eigenvalues of (3.16) are $\omega_{1},-\omega_{1}, \omega_{2},-\omega_{2}$ where

$$
\begin{aligned}
& \omega_{1}^{2}=R+S \\
& \omega_{2}^{2}=R-S
\end{aligned}
$$

with

$$
R=\frac{\left(a^{2}+1-2 \alpha(a A-1)\right)}{2}
$$




$$
S=\frac{\sqrt{\Delta}}{2}
$$

and

$$
\Delta=\left(a^{2}+1-2 \alpha(a A-1)\right)^{2}-4\left(a^{2}-2 a \alpha(A-a)\right) .
$$

Imposing symmetry and $u^{\prime}(0)=0$, the general solution of ODE(3.16) can be written in the form:

$$
u_{\mathrm{I}}(x)=C\left(e^{\omega_{1} x}+e^{-\omega_{1} x}\right)+D\left(e^{\omega_{2} x}+e^{-\omega_{2} x}\right)+U_{0}
$$

where

$$
U_{0}=\frac{2(A-a)\left(\beta-\alpha u_{T}\right)}{a-2 \alpha(A-a)}
$$

for $x \in\left(-x_{T}, x_{T}\right), x_{T} \in \Re, C, D \in \mathbf{C}$, and $u_{\mathrm{I}}(x) \in \Re$.

The single-pulse solutions of (3.6) are found by matching $u_{I}, u_{I I}$, and $u_{I I I}$ across $x_{T}$ and $-x_{T}$ using the matching conditions (3.9) - (3.10). We investigate the existence and shape of single-pulse solutions as we change the gain and connection function. For simplicity, we call $x_{T}$ the width of a pulse although it is actually the half width. The height of a single-pulse is $u(0)$. The firing rate of the pulse is given by $f[u]$.

3.2. Solutions for the Amari case $(\alpha=0)$. Amari found conditions for which single-pulse solutions exist for (2.4) with general Mexican Hat connectivity and the Heaviside gain function [3. Here, we revisit the Amari case for the exponential connection function (2.1). When $\alpha=0$ and $\beta=1$, the gain function (2.2) becomes the Heaviside function $\Theta(u)$ and the term $2(a A-1) \frac{d^{2} f[u]}{d x^{2}}$ does not exist in ODE (3.7). The eigenvalues (3.17) and (3.18) become simple and the solutions (3.22) and (3.14) are

$$
\begin{aligned}
u_{\mathrm{I}}(x) & =C\left(e^{a x}+e^{-a x}\right)+D\left(e^{x}+e^{-x}\right)+U_{0}, \\
u_{\mathrm{II}}(x) & =E e^{-a x}+F e^{-x},
\end{aligned}
$$

respectively. Applying conditions (3.9)-(3.13) to (3.23) and (3.24) yields the system

$$
\begin{gathered}
E e^{-a x_{T}}+F e^{-x_{T}}=u_{T} \\
C\left(e^{a x_{T}}+e^{-a x_{T}}\right)+D\left(e^{x_{T}}+e^{-x_{T}}\right)+\frac{2(A-a) \beta}{a}=u_{T} \\
a C\left(e^{a x_{T}}-e^{-a x_{T}}\right)+D\left(e^{x_{T}}-e^{-x_{T}}\right)=-a E e^{-a x_{T}}-F e^{-x_{T}} \\
a^{2} C\left(e^{a x_{T}}+e^{-a x_{T}}\right)+D\left(e^{x_{T}}+e^{-x_{T}}\right)=a^{2} E e^{-a x_{T}}+F e^{-x_{T}}-2(a A-1) \beta \\
a^{3} C\left(e^{a x_{T}}-e^{-a x_{T}}\right)+D\left(e^{x_{T}}-e^{-x_{T}}\right)=-a^{3} E e^{-a x_{T}}-F e^{-x_{T}}
\end{gathered}
$$

The system (3.25) - (3.29) is linear in the coefficients $C, D, E$, and $F$ which can be solved in terms of $x_{T}$ :

$$
\begin{aligned}
C & =-\frac{A}{a} e^{-a x_{T}} \\
D & =e^{-x_{T}} \\
E & =\frac{A}{a}\left(e^{a x_{T}}-e^{-a x_{T}}\right) \\
F & =-\left(e^{x_{T}}-e^{-x_{T}}\right)
\end{aligned}
$$



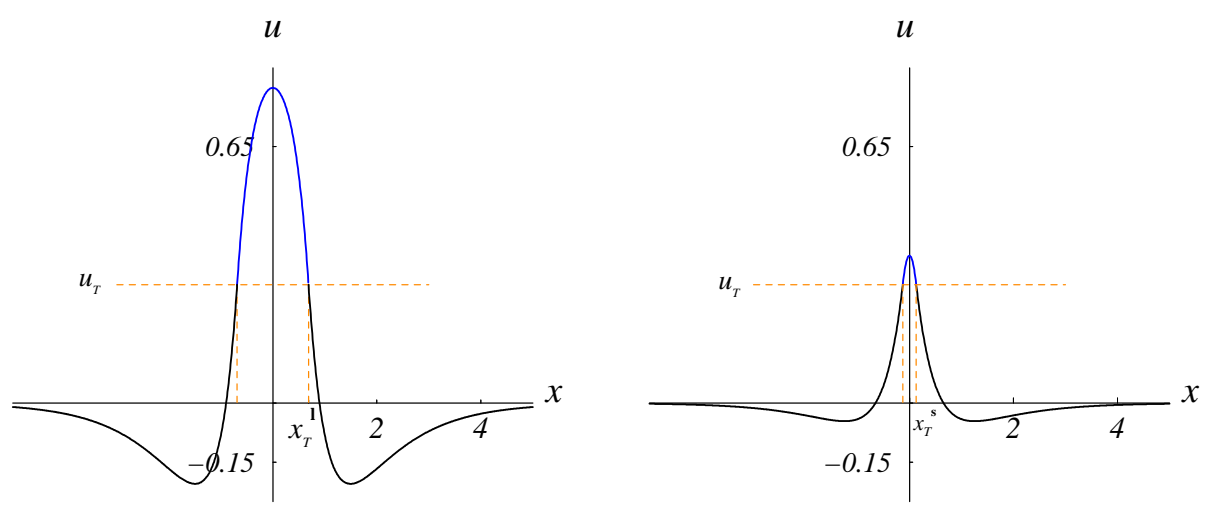

FIG. 3.2. Large single-pulse $\mathbf{l}$ and small single-pulse $\mathbf{s}$ for $A=2.8, a=2.6, \alpha=0$, $u_{T}=0.3$. (Left) Single-pulse 1: $x_{T}^{1}=0.68633$, height $=u(0)=0.79991$. (Right) Single-pulse s: $x_{T}^{\mathbf{s}}=0.12985$, height $=u(0)=0.37358$.

¿From these coefficients we arrive at the following proposition for single-pulse solutions.

Proposition 3.3. There are two pulse solutions when $u_{T} \leq \int_{0}^{(\ln A) /(a-1)} w(x) d x$ and $\left(\frac{A}{a}-1\right)<u_{T}$ for $A>a$ and $0 \leq u_{T}$ for $A<a$.

Proof. Substituting $E$ and $F$ into (3.25) (or $C$ and $D$ into (3.26), gives an existence condition for a single-pulse: $\Phi\left(x_{T}\right)=u_{T}$, where

$$
\Phi(x)=\frac{A}{a}\left(1-e^{-2 a x}\right)-\left(1-e^{-2 x}\right) .
$$

We term $\Phi(x)$ the "existence function". Two examples are shown in Figs. 3.3 and 3.4 where the curve $\Phi(x)$ crosses $u_{T}$ twice, implying that there are two single-pulse solutions. Since

$$
\lim _{x \rightarrow \infty} \Phi(x)=\frac{A}{a}-1=\left\{\begin{array}{lll}
<0 & \text { if } A<a \quad \text { (Figure 3.3) } \\
\geq 0 & \text { if } A \geq a \quad \text { (Figure 3.4) }
\end{array}\right.
$$

the lower bound of $u_{T}$ that supports two pulses is 0 if $A<a$ and the lower bound of $u_{T}$ that guarantees two pulses is $\frac{A}{a}-1$ when $A>a$.

The upper bound on threshold $u_{T}$ that supports two pulse solutions is the maximum of $\Phi(x)$. Solving

$$
\frac{d \Phi}{d x}=A e^{-2 a x}-2 e^{-2 x}=0
$$

gives $x=\frac{\ln A}{2(a-1)}$. Thus $\Phi$ reaches its maximum at

$$
\Phi(x)=\frac{A}{a}\left(1-e^{-\frac{a \ln A}{a-1}}\right)-\left(1-e^{-\frac{\ln A}{a-1}}\right)=\int_{0}^{\frac{\ln A}{a-1}} w(x) d x
$$

proving the proposition.

Proposition 3.3 does not immediately imply that there are two single-pulses because for a small threshold there can exist a dimple-pulse (Fig 3.5]) In Fig. 3.4 as 
$\Phi$

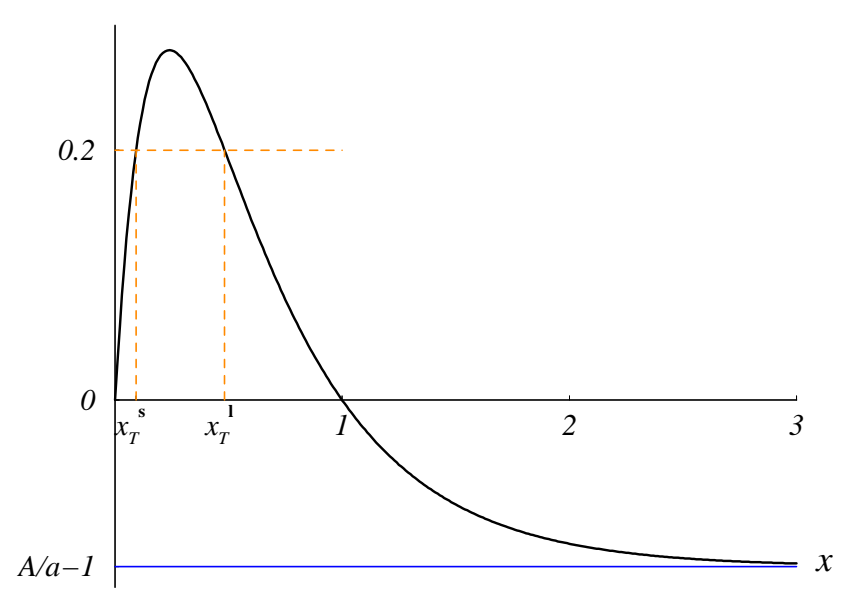

Fig. 3.3. Existence function $\Phi(x)$ when $A<a$ with $\alpha=0, A=2.6, a=3$. $\lim _{x \rightarrow \infty} \Phi(x)=\frac{A}{a}-1=-0.1333 . \Phi(x)$ gives the range of thresholds $u_{T}$ that supports two single-pulse solutions. Example: at $u_{T}=0.2, \Phi(x)$ shows that we have a single-pulse solution $\mathbf{l}$ with width $x_{T}^{1}$; the second single-pulse solution $\mathbf{s}$ is narrower and has width $x_{T}^{\mathbf{s}}$.

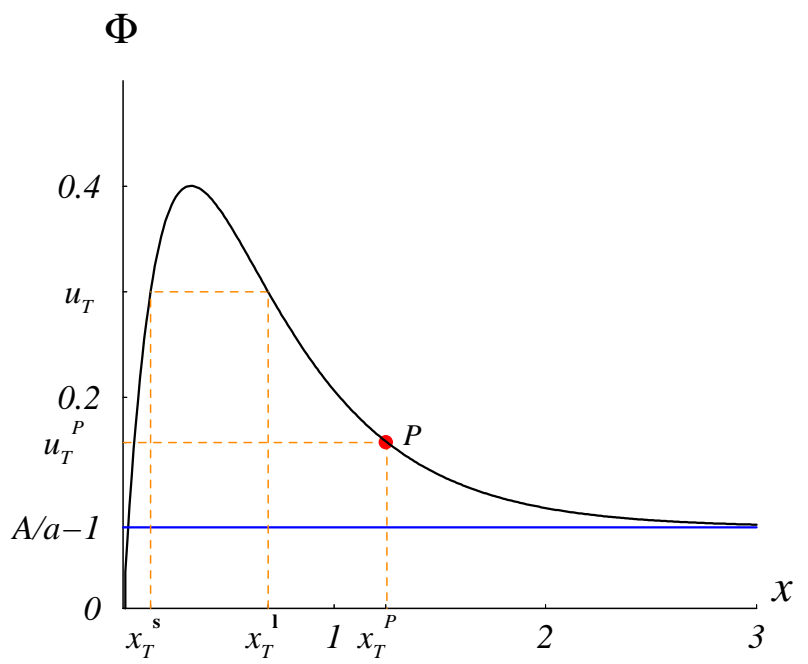

FIG. 3.4. Existence function $\Phi(x)$ when $A>a$. $\alpha=0, A=2.8, a=2.6$, $\lim _{x \rightarrow \infty} \Phi(x)=\frac{A}{a}-1=0.07692$. Example: at $u_{T}=0.3, \Phi(x)$ shows that there is a wide singlepulse solution $\mathbf{l}$ with width $x_{T}^{1}=0.68633$ and a narrower single-pulse solution $\mathbf{s}$ with width $x_{T}^{\mathbf{s}}=0.12985 . P$ is the transition point where single-pulse $\mathbf{l}$ changes into a dimple-pulse $\mathbf{d}$. At the transition, $u_{T}^{P}=0.15672$ and $x_{T}^{P}=1.24379$.

$u_{T}$ is lowered, $P$ is the transition point where $u^{\prime \prime}=0$ and single-pulse $\mathbf{l}$ transforms into the dimple-pulse $\mathbf{d}$. The small single-pulse $\mathbf{s}$ always remains a single-pulse. The transition point $P$ is identified by following $u^{\prime \prime}(0)$ as a function of $u_{T}$ using the continuation program AUTO. In the accompanying paper we compute the stability of these solutions. In agreement with Amari [3] we find that the large pulse is stable and the 


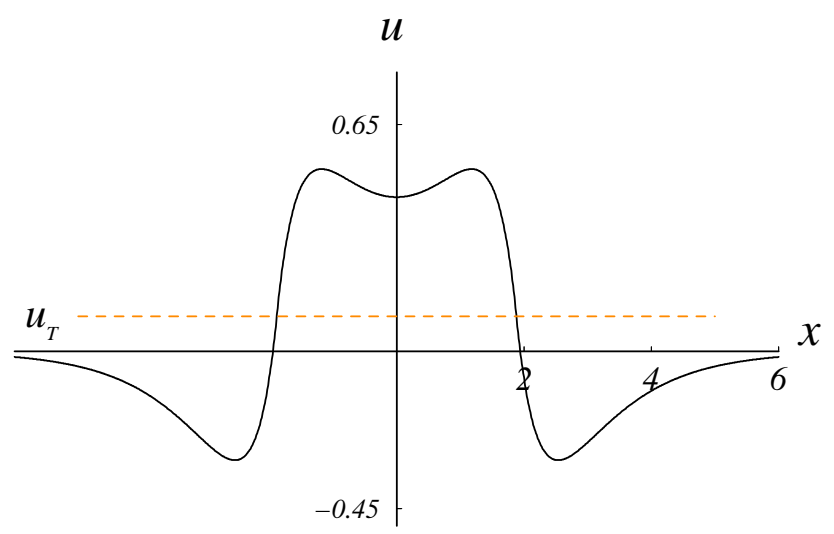

Fig. 3.5. Dimple-pulse $\mathbf{d}$ for $A=2.8, a=2.6, \alpha=0, u_{T}$ with width $x_{T}^{\mathrm{d}}=1.8832$.

small pulse is unstable. Additionally, we find that dimple pulse solutions can also be stable.

3.3. Solutions for the general case. For the general case of $\alpha \neq 0$, the complex eigenvalues $\omega_{1},-\omega_{1}, \omega_{2},-\omega_{2}$ given by (3.17) and (3.18) will change form for different parameter values. The transition points for the eigenvalues are given by the relative signs of functions $R$ (3.19), $S$ (3.20) and $\Delta$ (3.21). There are three cases: both eigenvalues $\omega_{1,2}$ are real, both are complex or both are imaginary.

We consider the transitions when $\alpha$ is changed for fixed $a$ and $A$. We find that there are five critical points where the eigenvalue structure changes. At $\alpha=\alpha_{0} \equiv$ $a /(2(A-a)), R-S=0$, with $R>0$ and $\Delta>0$. The solutions of the quadratic equation $\Delta(\alpha)=0$ give $\alpha_{1}$ and $\alpha_{3}$. At $\alpha_{2}, R=0$. At $\alpha_{4}=a /(2(A-a)), R+S=0$ with $R<0$ and $\Delta>0$. We arrange $\alpha_{i}(i=0,1,2,3,4)$ in increasing order. $\omega_{1}$ and $\omega_{2}$ are complex conjugates for both $\alpha \in\left(\alpha_{1}, \alpha_{2}\right)$ and $\alpha \in\left(\alpha_{2}, \alpha_{3}\right)$. In our analysis, we only consider the case where $\alpha>0$ (i.e. the firing rate is increasing with input). The case of $\alpha=0$ with the general connection weight function was fully treated in [3] and is reevaluated in Sec. 3.2 Tables 3.13 .2 and 3.3 enumerate all the possible forms of $\omega_{1}$ and $\omega_{2}$.

TABLE 3.1

Eigenvalue chart when $A>a$

\begin{tabular}{|c|c|c|c|c|c|c|}
\hline & E1 & E2 & E3 & E4 & E5 & E6 \\
\hline & $\triangle>0$ & $\triangle>0$ & $\triangle>0$ & $\triangle=0$ & $\triangle<0$ & $\Delta>0$ \\
& $R<0<|R|$ & & $R<0<S$ & & & $R<0<|R|$ \\
& $|R|<S$ & $0<S<R$ & $S<|R|$ & & & $|R|=S$ \\
\hline$\omega_{1}$ & real & real & imaginary & $=\omega_{2}$ & $=\omega_{2}^{*}$, complex & $=\sqrt{2 R}$ \\
\hline$\omega_{2}$ & imaginary & real & imaginary & $=\omega_{1}$ & $=\omega_{1}^{*}$, complex & 0 \\
\hline$\alpha$ & $\left(\alpha_{4}, \infty\right)$ & $\left(-\infty, \alpha_{1}\right)$ & $\left(\alpha_{3}, \alpha_{4}\right)$ & $\alpha_{1}, \alpha_{3}$ & $\left(\alpha_{1}, \alpha_{3}\right)$ & $\alpha_{4}$ \\
\hline
\end{tabular}

Although both $\alpha_{0}$ and $\alpha_{4}$ have the same expression, they do not co-exist. When $A<a, \alpha_{0}=\frac{a}{2(A-a)}<0$ and when $A>a, \alpha_{4}=\frac{a}{2(A-a)}>0$. For all values of $\omega_{1}$ and $\omega_{2}, u_{\mathrm{II}}(x)$ and $u_{\mathrm{III}}(x)$ always have the form (3.14) and (3.15) respectively. 
TABLE 3.2

Eigenvalue chart when $A<a$.

\begin{tabular}{|c|c|c|c|c|c|c|}
\hline & E1 & E2 & E3 & E4 & E5 & E6 \\
\hline & $\triangle>0$ & $\triangle>0$ & $\triangle>0$ & $\triangle=0$ & $\triangle<0$ & $\Delta>0$ \\
& $0<R<S$ & $0<S<R$ & $R<0<S<|R|$ & & & $0<R=S$ \\
\hline$\omega_{1}$ & real & real & imaginary & $=\omega_{2}$ & complex & $=\sqrt{2 R}$ \\
\hline$\omega_{2}$ & imaginary & real & imaginary & $=\omega_{1}$ &, complex & 0 \\
\hline$\alpha$ & $\left(-\infty, \alpha_{0}\right)$ & $\left(\alpha_{0}, \alpha_{1}\right)$ & $\left(\alpha_{3}, \infty\right)$ & $\alpha_{1}, \alpha_{3}$ & $\left(\alpha_{1}, \alpha_{3}\right)$ & $\alpha_{0}$ \\
\hline
\end{tabular}

TABLE 3.3

Eigenvalue chart when $A=a$.

\begin{tabular}{|c|c|c|c|c|c|c|}
\hline & E1 & $\mathrm{E} 2$ & E3 & E4 & E5 & E6 \\
\hline & $\begin{array}{c}\triangle>0 \\
0<R<S\end{array}$ & $\begin{array}{c}\triangle>0 \\
0<S<R\end{array}$ & $\begin{array}{c}\triangle>0 \\
R<0<S<|R|\end{array}$ & $\triangle=0$ & $\triangle<0$ & $R=S$ \\
\hline$\omega_{1}$ & \multirow{3}{*}{$\emptyset$} & real & imaginary & $=\omega_{2}$ & $=\omega_{2}^{*}$, complex & \multirow{3}{*}{$\emptyset$} \\
\hline$\omega_{1}$ & & real & imaginary & $=\omega_{2}$ & $=\omega_{1}^{*}$, complex & \\
\hline$\alpha$ & & $\left(-\infty, \alpha_{1}\right)$ & $\left(\alpha_{3}, \infty\right)$ & $\alpha_{1}, \alpha_{3}$ & $\left(\alpha_{1}, \alpha_{3}\right)$ & \\
\hline
\end{tabular}

\subsection{Solutions for real eigenvalues.}

3.4.1. Construction of single-pulse solutions. For $\alpha \in\left(0, \alpha_{1}\right)$, both $\Delta$ and $R$ are positive, so $\omega_{1}$ and $\omega_{2}$ are both real. Hence $u_{\mathrm{I}}(x)$ and $u_{\mathrm{II}}(x)$ have the following form:

$$
\begin{aligned}
u_{\mathrm{I}}(x) & =C\left(e^{\omega_{1} x}+e^{-\omega_{1} x}\right)+D\left(e^{\omega_{2} x}+e^{-\omega_{2} x}\right)+\frac{2(A-a)\left(\beta-\alpha u_{T}\right)}{a-2 \alpha(A-a)} \\
u_{\mathrm{II}}(x) & =E e^{-a x}+F e^{-x}
\end{aligned}
$$

When eigenvalues $\omega_{1}$ and $\omega_{2}$ are real, $C$ and $D$ must also be real for real $u_{\mathrm{I}}(x)$. Applying the the matching conditions (3.9)-(3.13) to (3.32) and (3.33) yields

$$
\begin{aligned}
E e^{-a x_{T}}+F e^{-x_{T}}= & u_{T} \\
C\left(e^{\omega_{1} x_{T}}+e^{-\omega_{1} x_{T}}\right)+D\left(e^{\omega_{2} x_{T}}+e^{-\omega_{2} x_{T}}\right)+U_{0}= & u_{T} \\
\omega_{1} C\left(e^{\omega_{1} x_{T}}-e^{-\omega_{1} x_{T}}\right)+\omega_{2} D\left(e^{\omega_{2} x_{T}}-e^{-\omega_{2} x_{T}}\right)= & -a E e^{-a x_{T}}-F e^{-x_{T}} \\
\omega_{1}^{2} C\left(e^{\omega_{1} x_{T}}+e^{-\omega_{1} x_{T}}\right)+\omega_{2}^{2} D\left(e^{\omega_{2} x_{T}}+e^{-\omega_{2} x_{T}}\right)= & a^{2} E e^{-a x_{T}}+F e^{-x_{T}}- \\
& 2(a A-1) \beta \\
\omega_{1}^{3} C\left(e^{\omega_{1} x_{T}}-e^{-\omega_{1} x_{T}}\right)+\omega_{2}^{3} D\left(e^{\omega_{2} x_{T}}-e^{-\omega_{2} x_{T}}\right)= & \left(-a^{3}+2 a \alpha(a A-1)\right) E e^{-a x_{T}}+ \\
& (-1+2 \alpha(a A-1)) F e^{-x_{T}}
\end{aligned}
$$

System (3.34) - (3.38) can be solved for the five unknowns $C, D, E, F$ and $x_{T}$ using Mathematica [74, giving an explicit formula for $u_{\mathrm{I}}(x)$ and $u_{\mathrm{II}}(x)$. The single-pulse is then given by $u_{\mathrm{I}}(x)$ on $\left(-x_{T}, x_{T}\right), u_{\mathrm{II}}(x)$ on $\left(x_{T}, \infty\right)$ and $u_{\mathrm{III}}(x)$ on $\left(-\infty, x_{T}\right)$. For the parameter set $\left(a, A, \alpha, \beta, u_{T}\right)=(2.6,2.8,0.15,1,0.400273)$, the solution is $\left(C, D, E, F, x_{T}\right)=(-0.8532,1.16865,2.94108,-0.89571,0.41902)$. Figure 3.6 shows a graph of this single-pulse. The height $u_{\mathrm{I}}(0)$ of the pulse is 0.77892 . Its width is $x_{T}^{1}=0.41902$. There also exists a second smaller and narrower single-pulse solution to (3.34)-(3.38) for the same set of parameters (see Fig. 3.7). The height and the width of this pulse are $u_{\mathrm{I}}(0)=0.6123$ and $x_{T}^{\mathrm{s}}=0.2582$ respectively. 


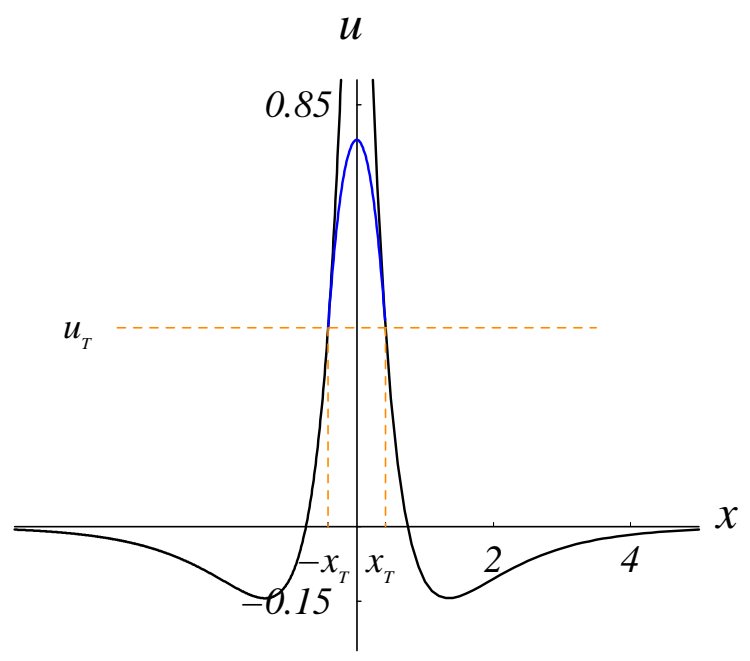

FIG. 3.6. Construction of large single-pulse 1. $A=2.8, a=2.6, \alpha=0.15, u_{T}=0.3$. $x_{T}^{1}=0.41092$, height $=u(0)=0.77892$.
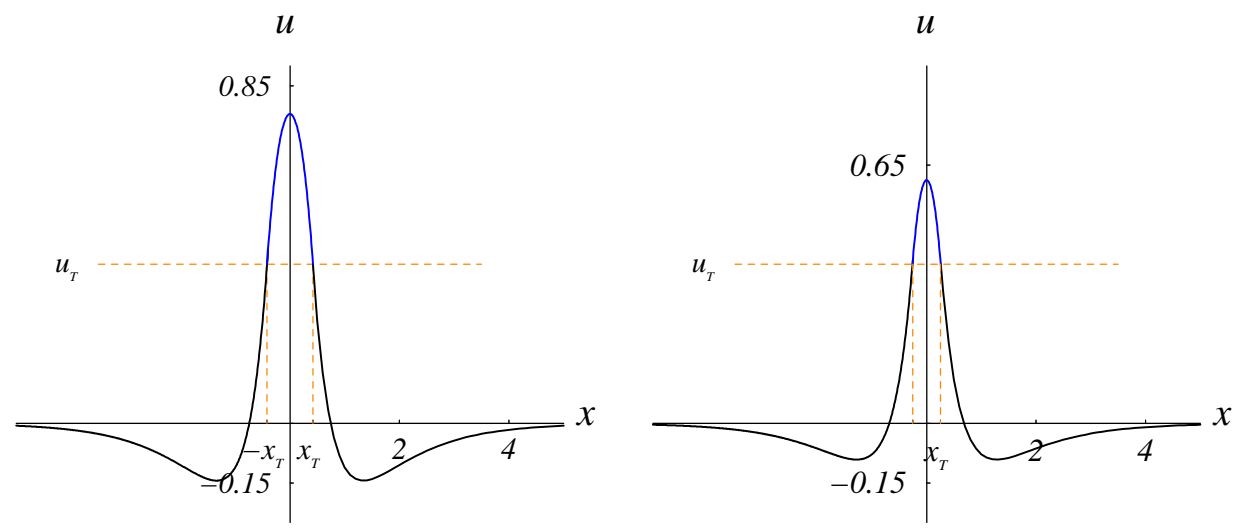

FIG. 3.7. Large single-pulse $\mathbf{l}$ and small single-pulse s. $A=2.8, a=2.6, \alpha=0.15$, $u_{T}=0.3$. (Left) Single-pulse 1: $x_{T}^{1}=0.41092$, height $=u(0)=0.77892$. (Right) Single-pulse $\mathrm{s}: x_{T}^{\mathbf{s}}=0.2582$, height $=u(0)=0.6123$.

When $\Delta=0$ and $R>0\left(\alpha=\alpha_{1}\right)$, there are repeating real eigenvalues: $\omega_{1}, \omega_{1}$, $-\omega_{1},-\omega_{1}$, where $\omega_{1}=\sqrt{R}$. By the symmetry of $u_{\mathrm{I}}(x), C 1=D 1$ and $C 2=D 2$, so $u_{\mathrm{I}}(x)$ from (3.22) can be written as

$$
u_{\mathrm{I}}(x)=C\left(e^{\omega_{1} x}+e^{-\omega_{1} x}\right)+D x\left(e^{\omega_{1} x}+e^{-\omega_{1} x}\right)+U_{0} .
$$

Applying matching conditions (3.9)-(3.13) gives a similar system to (3.34)-(3.38) to which solutions can be found numerically.

3.4.2. Finding solutions for real eigenvalues using the existence function. In order to perform numerical continuation on the single-pulse solutions, it is more convenient to utilize the existence function $\Phi(x)$ introduced by Amari [3] and calculated in Sec. 3.2 We compute it for the general case by first eliminating the threshold $u_{T}$ from system (3.34)-(3.38) to get an equivalent four equation system

$$
C\left(e^{\omega_{1} x_{T}}+e^{-\omega_{1} x_{T}}\right)+D\left(e^{\omega_{2} x_{T}}+e^{-\omega_{2} x_{T}}\right)+U_{0}=
$$




$$
\begin{aligned}
& \frac{a}{a-2 \alpha(A-a)}\left(E e^{-a x_{T}}+F e^{-x_{T}}\right) \\
& \omega_{1} C\left(e^{\omega_{1} x_{T}}-e^{-\omega_{1} x_{T}}\right)+\omega_{2} D\left(e^{\omega_{2} x_{T}}-e^{-\omega_{2} x_{T}}\right)=-a E e^{-a x_{T}}-F e^{-x_{T}} \\
& \omega_{1}^{2} C\left(e^{\omega_{1} x_{T}}+e^{-\omega_{1} x_{T}}\right)+\omega_{2}^{2} D\left(e^{\omega_{2} x_{T}}+e^{-\omega_{2} x_{T}}\right)= a^{2} E e^{-a x_{T}}+F e^{-x_{T}}- \\
& 2(a A-1) \beta \\
& \omega_{1}^{3} C\left(e^{\omega_{1} x_{T}}-e^{-\omega_{1} x_{T}}\right)+\omega_{2}^{3} D\left(e^{\omega_{2} x_{T}}-e^{-\omega_{2} x_{T}}\right)=\left(-a^{3}+2 a \alpha(a A-1)\right) E e^{-a x_{T}}+ \\
&(-1+2 \alpha(a A-1)) F e^{-x_{T}}
\end{aligned}
$$

Equations (3.40)-(3.44) form a linear system in $C, D, E$, and $F$. To obtain an existence function $\Phi(x)$, we construct coefficient vectors

$$
\begin{aligned}
& m_{1}=\left(\begin{array}{c}
e^{\omega_{1} x_{T}}+e^{-\omega_{1} x_{T}} \\
\omega_{1}\left(e^{\omega_{1} x_{T}}-e^{-\omega_{1} x_{T}}\right) \\
\omega_{1}^{2}\left(e^{\omega_{1} x_{T}}+e^{-\omega_{1} x_{T}}\right) \\
\omega_{1}^{3}\left(e^{\omega_{1} x_{T}}-e^{-\omega_{1} x_{T}}\right)
\end{array}\right), m_{2}=\left(\begin{array}{c}
e^{\omega_{2} x_{T}}+e^{-\omega_{2} x_{T}} \\
\omega_{2}\left(e^{\omega_{2} x_{T}}-e^{-\omega_{2} x_{T}}\right) \\
\omega_{2}^{2}\left(e^{\omega_{2} x_{T}}+e^{-\omega_{2} x_{T}}\right) \\
\omega_{2}^{3}\left(e^{\omega_{2} x_{T}}-e^{-\omega_{2} x_{T}}\right)
\end{array}\right), \\
& m_{3}=\left(\begin{array}{c}
a \\
\frac{a}{a-2 \alpha(A-a)} \\
a \\
-a^{2} \\
a-2 \alpha(A-a) \\
1 \\
-1 \\
a^{3}-2 a \alpha(a A-1)
\end{array}\right), m_{4}=\left(\begin{array}{c} 
\\
1-2 \alpha A-1)
\end{array}\right), \\
& m_{0}=\left(\begin{array}{c}
(A-a) \beta \\
a-2 \alpha(A-a) \\
0 \\
-2(a A-1) \beta \\
0
\end{array}\right) .
\end{aligned}
$$

Let $D E T_{x_{T}}(\alpha)=\mid \begin{array}{lllll}m_{1} & m_{2} & m_{3} & m_{4} & \text { |, where |.| is the determinant. For pa- }\end{array}$ rameters $\left(a, A, \alpha, \beta, u_{T}\right)=(2.6,2.8,0.15,1,0.400273)$, the solution $\left(C, D, E, F, x_{T}\right)=$ $(-0.8532,1.16865,2.94108,-0.89571,0.41902)$ with $D E T_{x_{T}}(\alpha)=-243.2415568475$ is given by Mathematica. We use this solution as an initial guess to continue system (3.34)-(3.38) using AUTO while following $D E T_{x_{T}}(\alpha)$ as $\alpha$ decreases to 0 and then increases to $\alpha_{1}$. The recorded value of $D E T_{x_{T}}$ shows that $D E T_{x_{T}}(\alpha) \neq 0$ as $\alpha<\alpha_{1}$. Therefore we can always solve the linear system (3.40)-(3.44) by Cramer's rule. The solutions for $E$ and $F$ given by

$$
\begin{aligned}
& E=\frac{\mid \begin{array}{llllll}
\mid & m_{1} & m_{2} & m_{0} & m_{4} & \mid
\end{array}}{\mid \begin{array}{llllll}
m_{1} & m_{2} & m_{3} & m_{4} & e^{-a x_{T}}
\end{array}}, \\
& F=\frac{\mid \begin{array}{llllll}
m_{1} & m_{2} & m_{3} & m_{0} & \mid
\end{array}}{\mid \begin{array}{llllll}
m_{1} & m_{2} & m_{3} & m_{4} & \mid e^{-x_{T}}
\end{array} .}
\end{aligned}
$$

are then substituted back into $u_{I I}(x)=E(x) e^{-a x}+F(x) e^{-x}$ to obtain the existence function

$$
\Phi(x)=E e^{-a x}+F e^{-x}=\frac{\left|\begin{array}{lllll}
m_{1} & m_{2} & m_{0} & \left(m_{3}-m_{4}\right)
\end{array}\right|}{\left|\begin{array}{lllll}
m_{1} & m_{2} & m_{3} & m_{4}
\end{array}\right|} .
$$

Figure 3.8 shows $\Phi(x)$ (3.44). $\Phi(x)$ approaches a limit as $x \rightarrow \infty$, but this limit is different from the limit of $\Phi(x)$ in the Amari case $(\alpha=0$.) 
Single-pulses of width $x_{T}$ are given by solutions of $\Phi\left(x_{T}\right)=u_{T}$. Since all four eigenvalues are real, there are no oscillations in $\Phi(x)$ and so there are at most two pulse solutions. One is a small single-pulse, and the other is either a large single-pulse or a dimple-pulse depending on the threshold $u_{T}$.

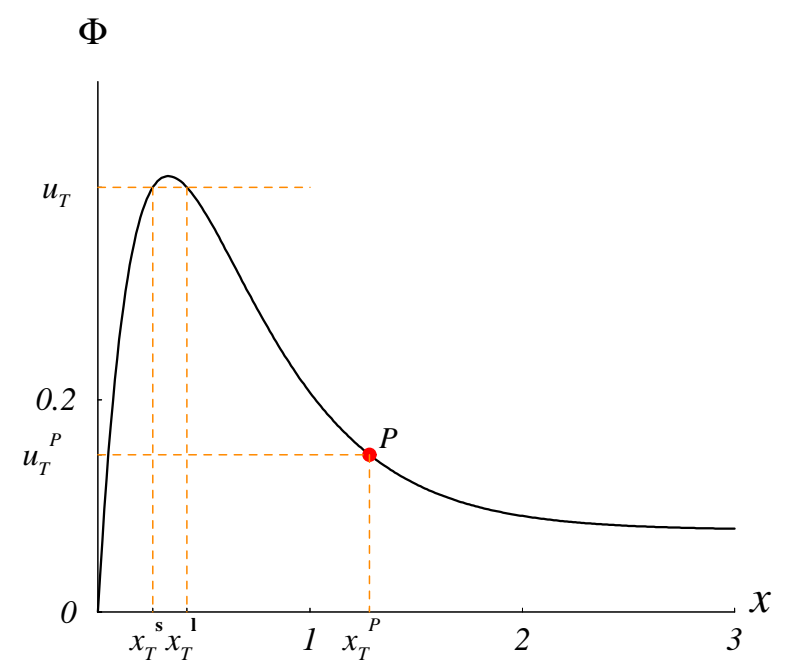

FIG. 3.8. Existence function $\Phi(x)$ for $\alpha=0.15, A=2.8, a=2.6$. At $u_{T}=0.400273$, $\Phi(x)$ has a single-pulse $\mathbf{l}$ which has width $x_{T}^{1}=0.4109$; the second single-pulse $\mathbf{s}$ is narrower with width $x_{T}^{\mathrm{s}}=0.2582$. At $P$, threshold $u_{T}^{P}=0.1489, u^{\prime \prime}(0)$ of the pulse at $P$ is 0 .

3.4.3. Transition point $P$ between single-pulses and dimple-pulses. The existence function $\Phi(x)$ gives a range of thresholds $u_{T}$ for which there exist two pulse solutions; a large pulse $\mathbf{l}$ (or dimple-pulse $\mathbf{d}$ ) and a small pulse $\mathbf{s}$, or only one small single-pulse solution. The $x$-value of the intersection of $u_{T}$ and $\Phi(x)$ is the width of a pulse. In figure 3.8 $x_{T}^{\mathrm{s}}$ is the width of $\mathbf{s}$, and $x_{T}^{1}$ is the width of $\mathbf{l}$. At $P$, the curvature at the peak of the pulse solution is zero (i.e. $u^{\prime \prime}(0)=0$ ) as seen in Fig. 3.10 For this set of parameters, dimple-pulses appear if $u_{T}$ is between $u_{T}^{P}=0.1489$ and $\lim _{x \rightarrow \infty} \Phi(x)$ (See Fig. 3.8). Figure 3.9] shows the continuation plot of $u^{\prime \prime}(0) ; u^{\prime \prime}(0)$ crosses zero at $u_{T}=u_{T}^{P}$.

3.4.4. Loss of existence for unbalanced synaptic connectivity. An examination of the shape of $\Phi(x)$ shows why single-pulses do not exist when excitation and inhibition are too much out of balance. When excitation dominates, $A / a>1$. For fixed $a, \alpha$ and threshold $u_{T}$, as $A$ becomes larger, the existence function $\Phi(x)$ moves up and $\lim _{x \rightarrow \infty} \Phi(x)$ becomes larger. The width of the large pulse $\mathbf{l}$ (or dimple-pulse) increases (Fig 3.11) When $\Phi(x)$ is tangent to $u_{T}$ for large $x$ (black curve in Fig 3.11) the width becomes $\infty$ and the pulse no longer exist. The pulse $\mathbf{l}$ or $\mathbf{d}$ can be regained by increasing $u_{T}$. However, for large enough $A / a, \Phi(x)$ will become monotonic (see Fig. (3.12) and only s exists.

When $A / a<1$, inhibition dominates excitation in the network. For fixed $a$ and $\alpha$, $\Phi(x)$ diminishes as $A$ is decreased (ratio $A / a$ becomes smaller.) Eventually, the ratio is small enough to make $\Phi(x)$ negative (see Fig. [3.13), and for $u_{T}>0$, single-pulses no longer exist. Inputs to the neurons in the network never exceed threshold so the neurons cannot fire. 


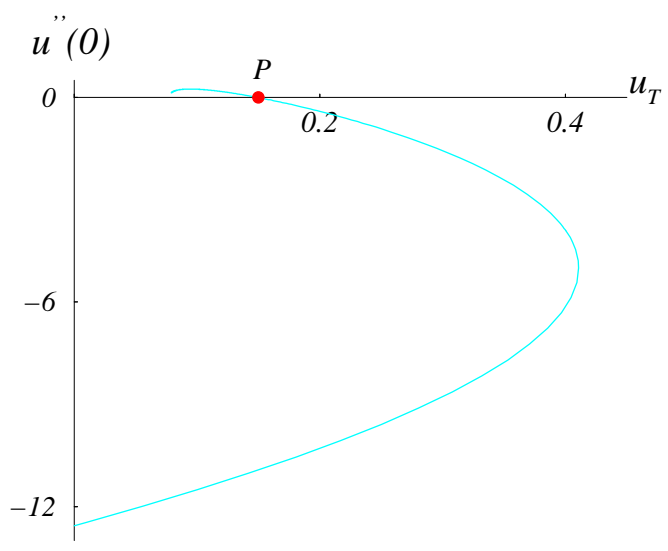

FIG. 3.9. Plot of $u^{\prime \prime}(0)$ when $\alpha=0.15, A=2.8, a=2.6 . \quad P$ is the transition point between single-pulse $\mathbf{l}$ and dimple-pulse $\mathbf{d}$. When threshold $u_{T}^{P}=0.1489, u^{\prime \prime}(0)=0$.

$u$

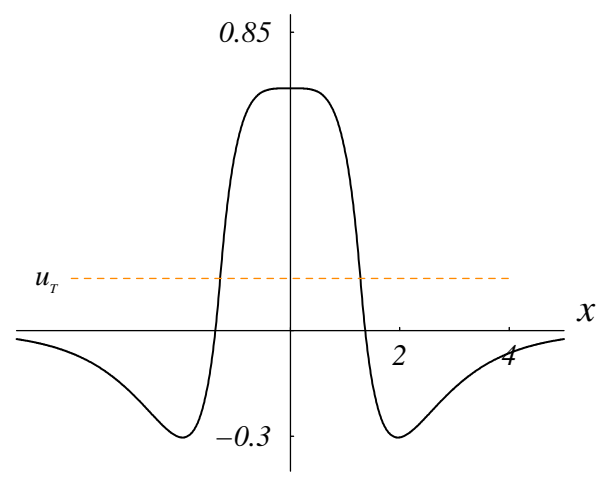

Fig. 3.10. Example of P-pulse with $a=2.6, A=2.8, \alpha=0.15, u_{T}^{P}=0.14838$. The width of this pulse is $x_{T}^{P}=1.27978$ and $u^{\prime \prime}(0)=0$.

$\Phi$

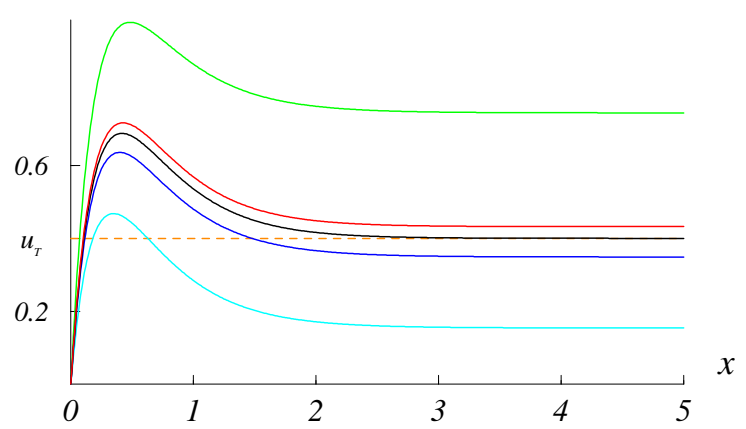

FIG. 3.11. $\Phi(x)$ with excitation dominating inhibition. $a=2.6, \alpha=0.05, u_{T}=0.400273$, and different values of $A$ : $A=3$ (cyan); $A=3.5$ (blue); $A=3.62$ (black); $A=3.7$ (red); $A=4.5$ (green). When $\Phi(x)$ is tangential to threshold $u_{T}$ for large $x$, the width of the large pulse is $\infty$. 


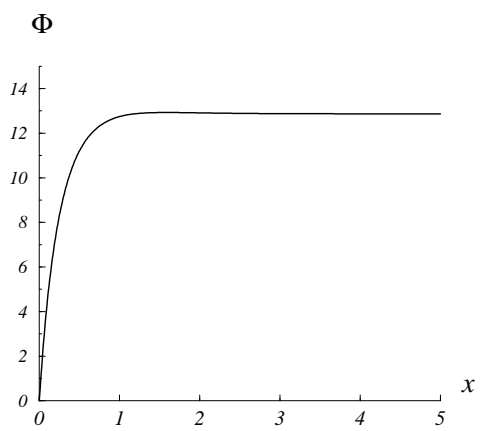

FIG. 3.12. $\Phi(x)$ with excitation dominating inhibition: $A=29.6, a=2.6, \alpha=0.03$. There exists only pulse $\mathbf{s}$, pulse $\mathbf{l}$ or $\mathbf{d}$ do not exist.

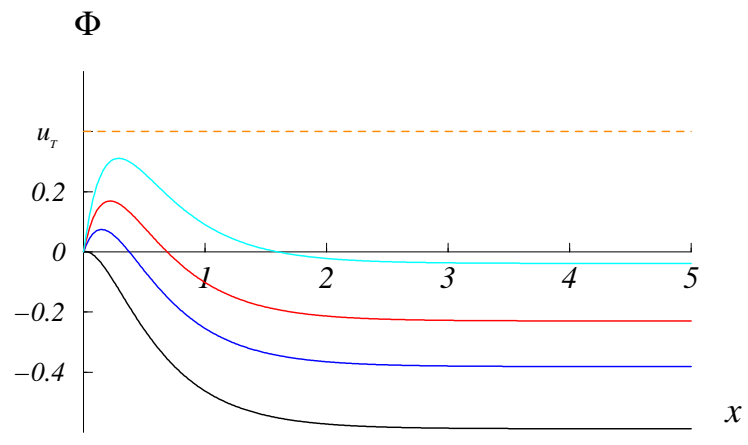

FIG. 3.13. $\Phi(x)$ with inhibition dominating excitation. $a=2.6, \alpha=0.05, u_{T}=0.400273$ and different values of $A$ : $A=2.5$ (cyan); $A=2$ (blue); $A=1.6$ (red); $A=1.05$ (black). There is neither pulse $\mathbf{s}$ nor pulse $\mathbf{l}$ for positive $u_{T}$ when $A / a$ is small enough (black color).

3.5. Solutions for complex eigenvalues. As seen in Tables 3.13 .2 and 3.3 the eigenvalues $\omega_{1}$ and $\omega_{2}$ form a complex conjugate pair for $\alpha_{1}<\alpha<\alpha_{3}$. Thus as long as $\alpha_{1}<\alpha_{3}$, complex eigenvalues can be found for arbitrary $a$ and $A$. Suppose $\omega_{1}=\omega_{2}^{*}=p+i q$. Then $p=\left(R^{2}+S^{2}\right)^{\frac{1}{4}} \cos \theta, p=\left(R^{2}+S^{2}\right)^{\frac{1}{4}} \sin \theta$, where $\theta=$ $\frac{1}{2} \arctan \frac{\sqrt{|\Delta|}}{2 R}$ for $\alpha \in\left(\alpha_{1}, \alpha_{2}\right)$ or $\theta=\frac{\pi}{2}+\frac{1}{2} \arctan \frac{\sqrt{|\Delta|}}{2 R}$ for $\alpha \in\left(\alpha_{2}, \alpha_{3}\right)$. When $\alpha>\alpha_{3}, \Delta>0, R<0$ and $S=\frac{\sqrt{\Delta}}{2}<|R|$. The real parts of $\omega_{1}$ and $\omega_{2}$ are both zero and $w_{1}=i q_{1}, w_{2}=i q_{2}$, where $q_{1}=\sqrt{|R+S|}$ and $q_{2}=\sqrt{|R-S|}$.

3.5.1. Construction of a single-pulse with complex eigenvalues. To ensure that $u_{\mathrm{I}}(x)$ is real, $C$ and $D$ must be complex. Imposing symmetry, gives $C=D^{*}$. Setting $C=C_{R}+i C_{I}$ implies $D=C_{R}-i C_{I}$. Substituting $C, D, \omega_{1}$ and $\omega_{2}$ into (3.22) for $u_{\mathrm{I}}(x)$ gives

$$
u_{\mathrm{I}}(x)=4 C_{R} \cos (q x) \cosh (p x)-4 C_{I} \sin (q x) \sinh (p x)+U_{0} .
$$

For simplicity, we relabel with $C=4 C_{R}$ and $D=-4 C_{I}$. When $\omega_{1}$ and $\omega_{2}$ are both imaginary, we have

$$
u_{\mathrm{I}}(x)=C \cos \left(q_{1} x_{T}\right)+D \cos \left(q_{2} x_{T}\right)+U_{0} .
$$

Applying the matching conditions (3.9) - (3.13), results in 5 algebraic equations with unknowns $C, D, E, F$ and $x_{T}$ which can be solved numerically to obtain the explicit 
form of $u_{\mathrm{I}}(x)$. The plots of pulses $\mathbf{l}$ and $\mathbf{s}$ are shown in Fig. 3.15

When $\alpha=\alpha_{3}, R<0$, implying $\omega_{1}=\omega_{2}=\sqrt{R}=i \sqrt{-R}$. Let $\omega=\sqrt{-R} \in \Re$. Then

$$
u_{\mathrm{I}}(x)=C_{1} \cos \omega x+C_{2} \sin \omega x+D_{1} x \cos \omega x+D_{2} x \sin \omega x+U_{0} .
$$

Since $u_{\mathrm{I}}(x)=u_{\mathrm{I}}(-x)$, then $C_{2}=D_{1}=0$ leaving

$$
u_{\mathrm{I}}(x)=C \cos \omega x+D x \sin \omega x+U_{0}
$$

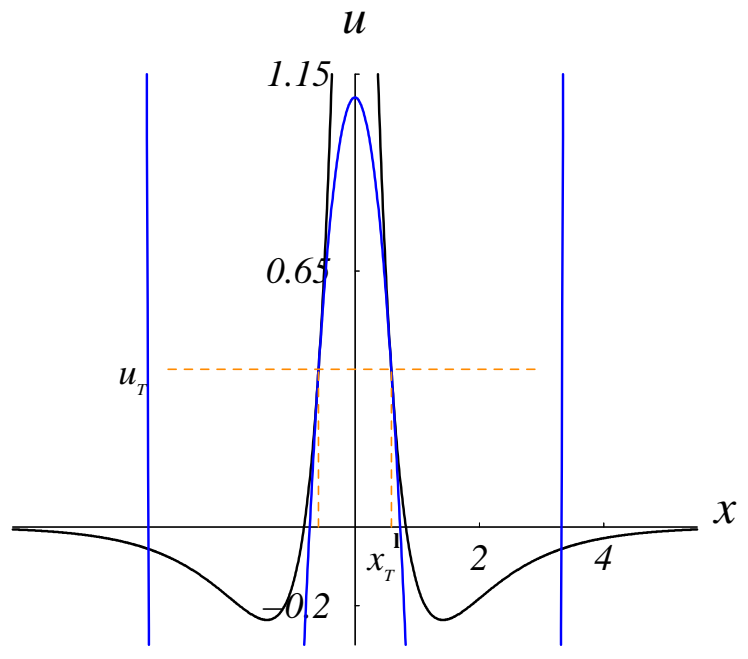

FIG. 3.14. Large single-pulse. $A=2.8, a=2.6, \alpha=0.6178, u_{T}=0.3, x_{T}^{1}=0.58384$, and $u(0)=1.0901$.
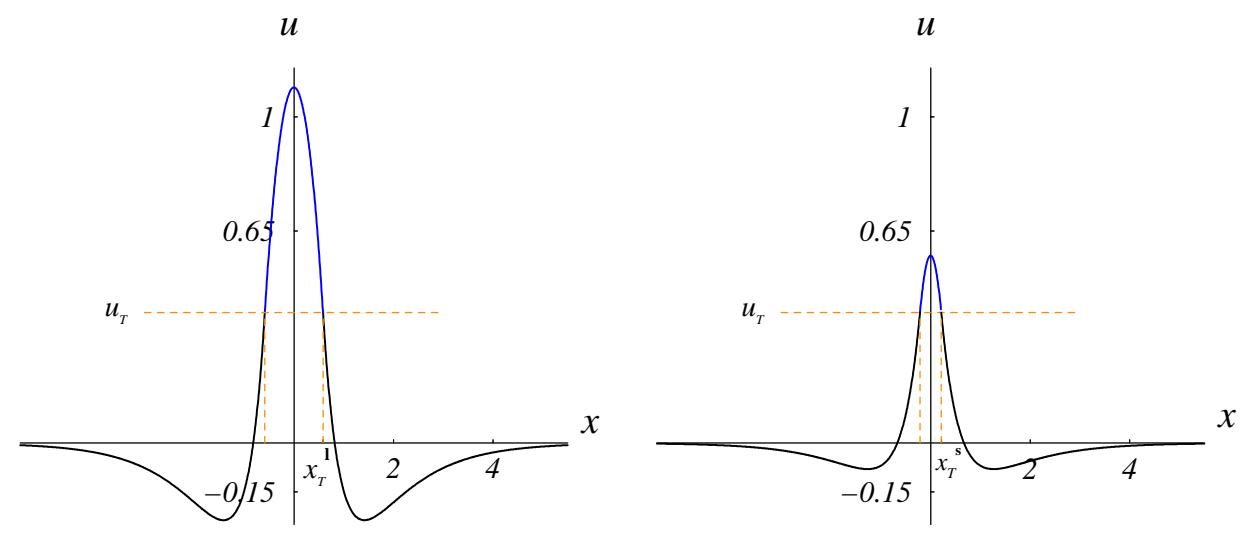

Fig. 3.15. Two single-pulses with $A=2.8, a=2.6, \alpha=0.6178, u_{T}=0.3$. (Left) Singlepulse $\mathbf{l}$ with $x_{T}^{1}=0.58384$ and $u(0)=1.0901$. (Right) Single-pulse $\mathbf{s}$ with $x_{T}^{\mathbf{s}}=0.21317$ and $u(0)=0.5744$.

3.5.2. Finding solutions for complex eigenvalues using the existence function. The existence function $\Phi(x)$ with complex $\omega_{1}$ and $\omega_{2}$ (Figs. 3.16 and 3.18) 
can be obtained using methods similar to those in Sec. 3.4.2. The main difference with the real case is that $\Phi(x)$ for complex eigenvalues can oscillate as seen in Fig. 3.16 After the first local minimum between $P_{1}$ and $P_{2}, \Phi(x)$ will approach a constant with decaying oscillations for increasing $x$. Additionally, if the threshold is between the first local minimum and the next local maximum, there exist more than two pulse solutions. Figure 3.17 shows an example where there is a small single-pulse and two dimple-pulses. There is never more than two coexisting pulses for real $\omega_{1}$ and $\omega_{2}$, which includes the Amari case, because the existence function $\Phi(x)$ does not oscillate. It is important to note that satisfying the existence condition $\Phi(x)=u_{T}$ is a necessary but not a sufficient condition for a pulse solution. It is possible to satisfy the matching conditions and not be a pulse. Thus although in principle, an infinite number of pulses could exist, in our experience, we find that most of the larger $x$ solutions are not pulses. As will be shown in the accompanying paper [33], pulse $\mathbf{s}$ is unstable and pulse $\mathbf{l}$ is stable. If there are three pulses, the largest third pulse which can be either a single pulse or a dimple pulse is unstable.

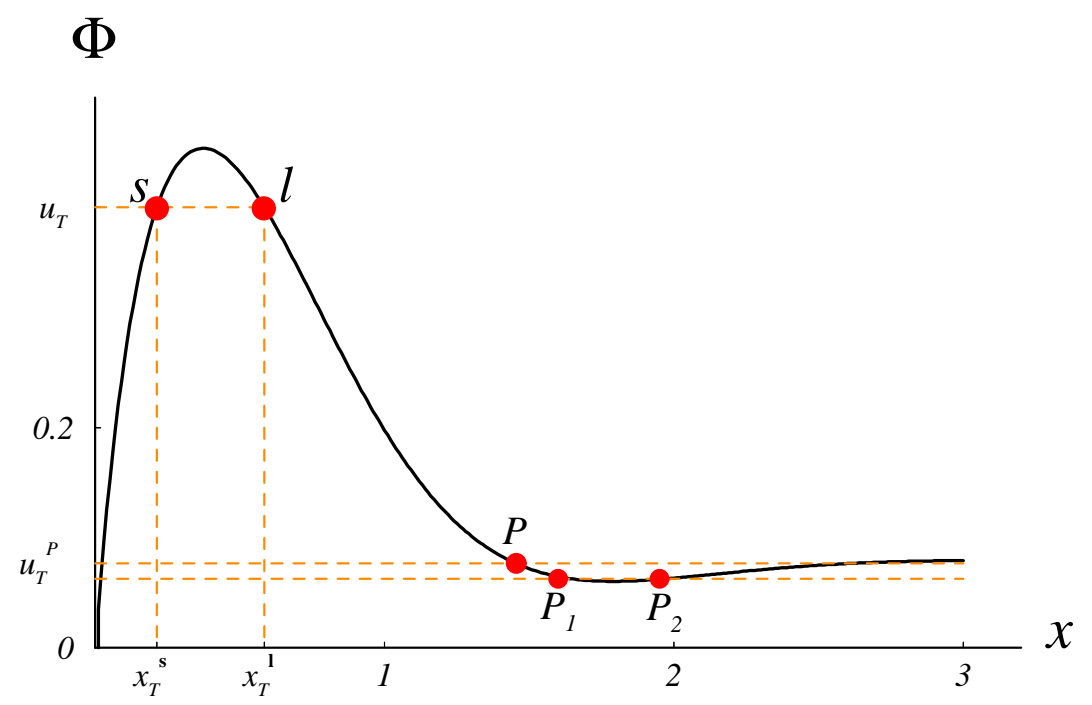

FIG. 3.16. Existence function $\Phi(x) . \alpha=0.6178, A=2.8, a=2.6$. At $u_{T}=0.400273$, $\Phi(x)$ shows that there is a single-pulse $\mathbf{l}$ which is wider and has width $x_{T}^{1}=0.58385$; the second single-pulse $\mathbf{s}$ is narrower and has width $x_{T}^{\mathrm{s}}=0.21317$. As we increase $u_{T}$ to the maximum of $\Phi(x)$, pulse $\mathbf{s}$ and $\mathbf{l}$ annihilate in a saddle-node bifurcation. At $P, u_{T}^{P}=0.0767$, $x_{T}^{P}=1.454, u^{\prime \prime}(0)=0$. At both $P_{1}$ and $P_{2}, u_{T}=0.063, u^{\prime \prime}(0)>0$, and the widths are 1.6 and 1.9 respectively. See Fig. 3.17 .

Oscillations in $\Phi(x)$ also exist when the eigenvalues are pure imaginary. As before, more than two pulses, including dimple-pulses, can coexist (see Fig. 3.18) depending on the threshold $u_{T}$. Fig. [3.18] shows a special case of a dimple-pulse where the dimple minimum reaches the threshold. If the minimum drops below the threshold, the dimple-pulse breaks into two disjointed single-pulses or a double-pulse. This doublepulse is not a valid solution because it violates the assumptions of the equations from which the solution was derived. However, double pulses can exist and we show this using a separate formalism in Sec. 5 .

3.5.3. Blow-up for large $\alpha$. For large enough $\alpha$, the large pulse $\mathbf{l}$ blows up at a critical value $\alpha^{0}$ and does not exist for $\alpha \geq \alpha_{0}$. The blow-up occurs in the regime 

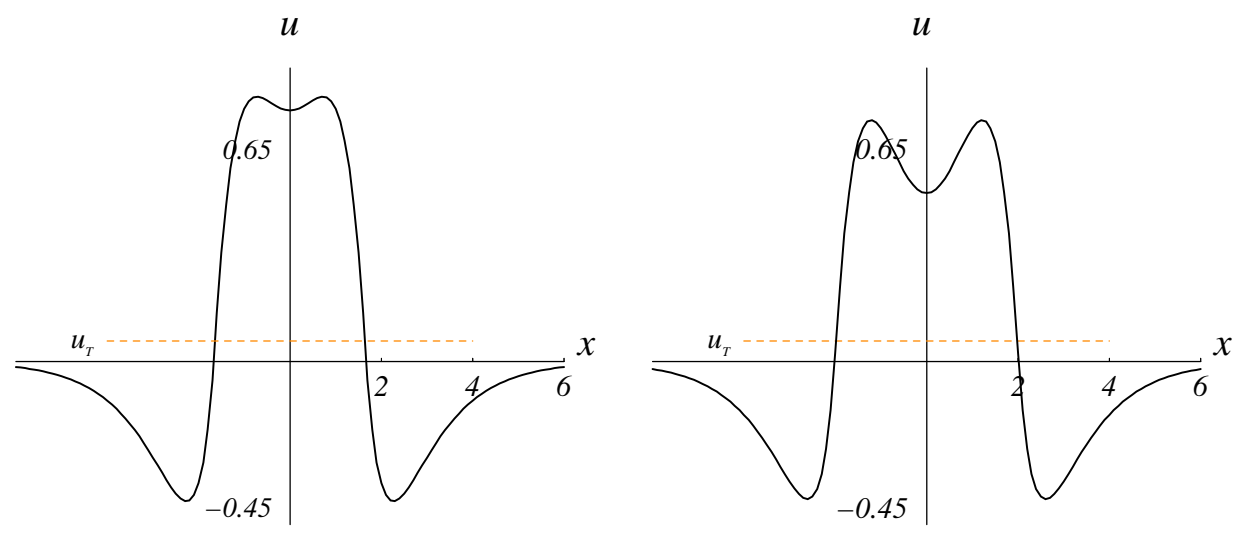

Fig. 3.17. Dimple-pulses with parameters $A=2.8, a=2.6, \alpha=0.6178, u_{T}=0.063$. (Left) Dimple-pulse at $P_{1}$ with $x_{T}^{\mathrm{d}}=1.6$. (Right) Dimple-pulse at $P_{2}$ with $x_{T}^{\mathrm{d}}=1.9$.

\section{$\Phi$}

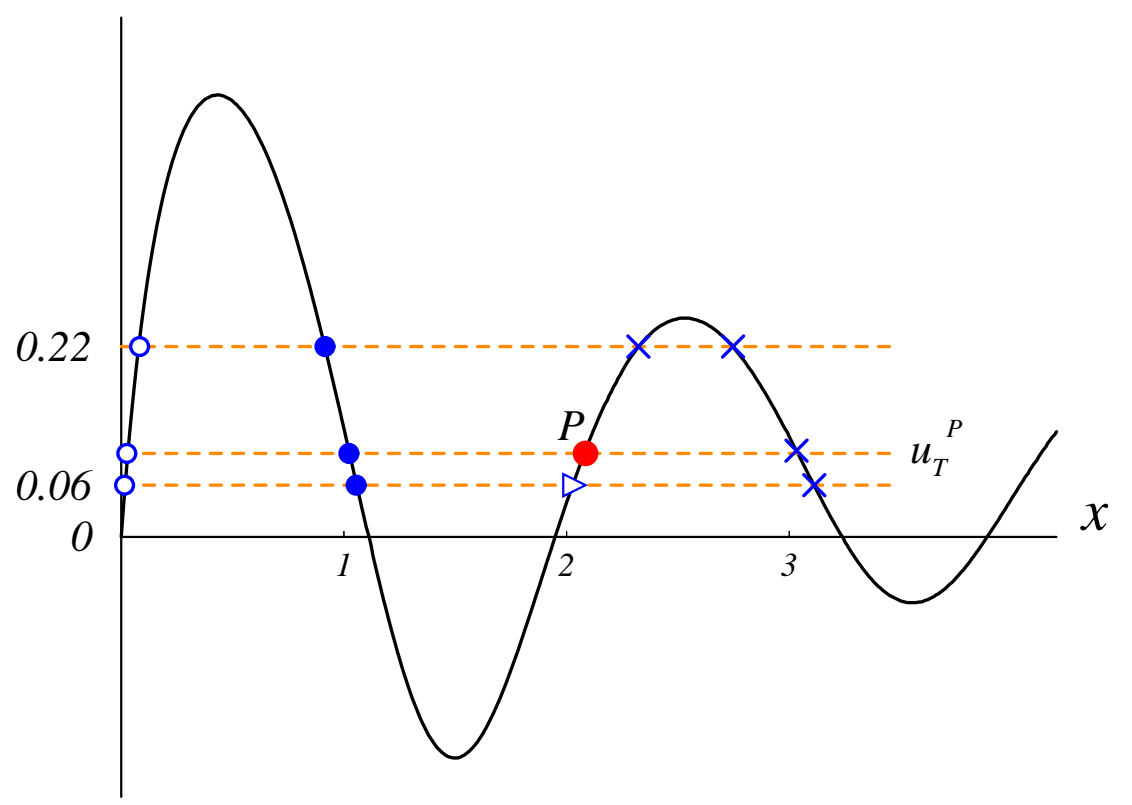

FIG. 3.18. Existence function $\Phi(x)$ with imaginary $\omega_{1}=\omega_{2}$ for $A=2.8, a=2.6, \alpha=\alpha_{3}$, $u_{T}^{P}=0.0967003$. The empty circles are small single-pulses. The solid circles are large singlepulses. The triangle is a dimple-pulse. Point $P$ is where the dimple-pulse (figure 3.19) breaks into a double-pulse. The $\times s$ are not valid solutions.

where both $\omega_{1}$ and $\omega_{2}$ are imaginary. Thus the height of pulse $\mathbf{l}$ is

$$
u(0)=C+D+\frac{2(A-a)\left(\beta-\alpha u_{T}\right)}{a-2 \alpha(A-a)},
$$

which can be expressed as

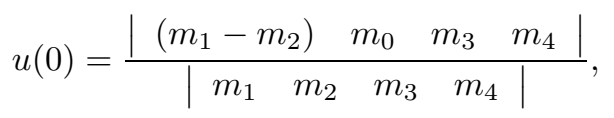




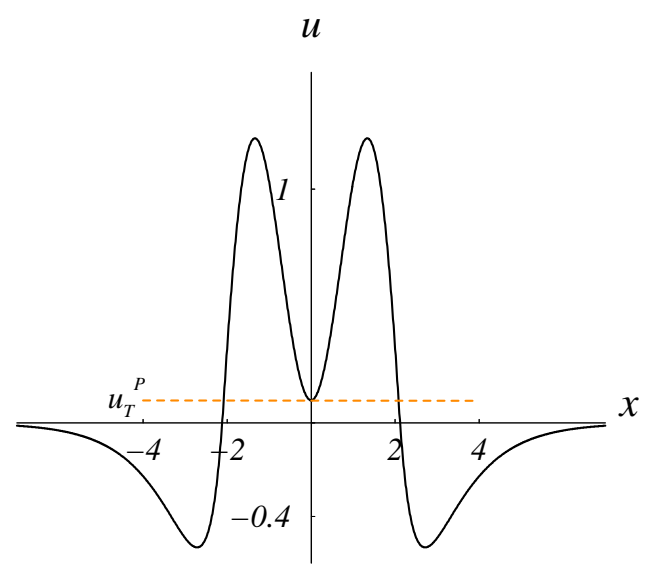

FIG. 3.19. The transition from a dimple-pulse to a double-pulse at $P$ with $u_{T}^{P}=0.0967003$, and $u(0)=0.0967003$.

where the coefficient factors $m_{1}, m_{2}, m_{3}, m_{4}$ for $C, D, E, F$ and $m_{0}$ are defined in section 3.4.2 The blow-up occurs because the denominator of (3.46) goes to zero at $\alpha=\alpha^{0}$ while the numerator remains finite, sending the height $u(0)$ of the large pulse to infinity.

We can also see the loss of $\mathbf{l}$ in the existence function:

$$
\Phi(x)=E e^{-a x}+F e^{-x}=\frac{\left|\begin{array}{llll}
m_{1} & m_{2} & m_{0} & \left(m_{3}-m_{4}\right)
\end{array}\right|}{\left|\begin{array}{llll}
m_{1} & m_{2} & m_{3} & m_{4}
\end{array}\right|} .
$$

Figure 3.20 shows that when $\alpha \geq \alpha^{0}$, there is always the small single-pulse $\mathbf{s}$ but no large single-pulse $\mathbf{l}$. A third solution (the third intersection of $u_{T}$ and $\Phi(x)$ ) could also exist but we have not examined this solution. As $\alpha$ increases, the height of $\mathbf{l}$ becomes very large but the width of the large pulse remains finite. This can be observed both from the existence function $\Phi(x)$ and the continuation plot (Fig 4.2) in Sec. 4.
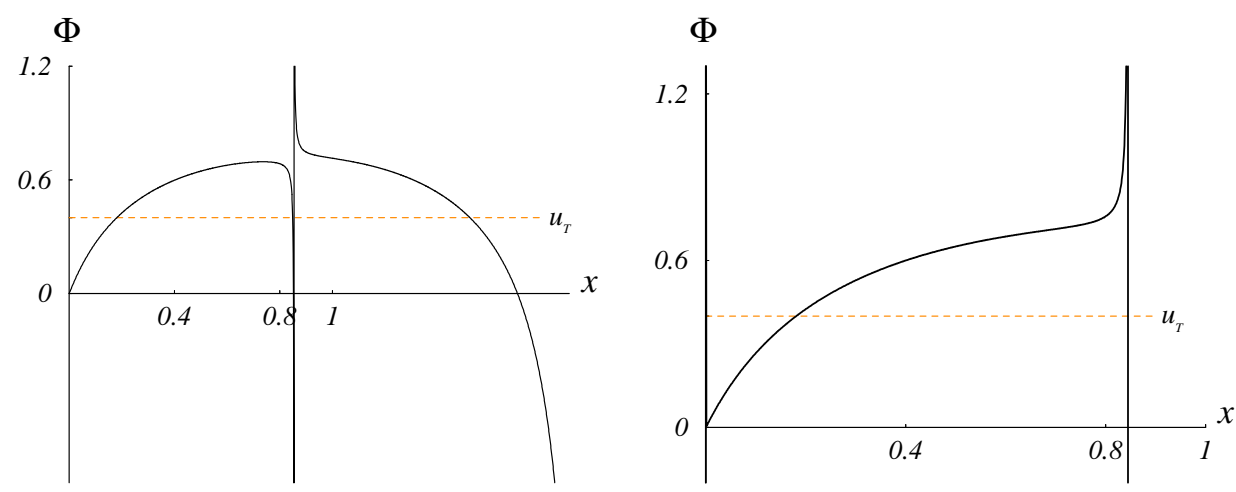

FIG. 3.20. Existence function $\Phi$ for imaginary $\omega_{1,2}$ with $A=2.8, a=2.6, u_{T}=0.400273$. (Left) $\alpha=1.4$. There is a single-pulse 1 , and $x_{T}^{1}=0.8491539857774331$, height $=u(0)=$ 146.2227855915919, which is big because $\alpha=1.4$ is close to $\alpha^{0}$ where DET $=0$. (Right) $\alpha=1.41>\alpha^{0}$. Single-pulse 1 no longer exists. The vertical line in both pictures is where $\Phi(x)$ blows up. 
4. Continuation in parameter space. One of our original goals was to understand how the shape of stationary pulses and their corresponding firing rates change as the parameters of synaptic connectivity and gain are changed. Here we give a global picture in the parameter space of $u_{T}, a, A$, and $\alpha$. A difficulty in this undertaking is that as the parameters are altered, the eigenvalue structure will make abrupt transitions. Hence, one must keep track of the eigenvalues and switch the form of the solutions when appropriate to construct a global picture.

As we saw before, the small and large pulses arise out of a saddle-node bifurcation. This gives a minimal condition for when pulses can exist. In Figs. 4.1 and 4.2 we show the large pulse $\mathbf{l}$ and the small pulse $\mathbf{s}$ arising from a saddle node bifurcation as $\alpha$ is increased for fixed $a$ and $A$. We have set the threshold to

$$
u_{T}^{0}=\int_{0}^{\ln A /(a-1)} w(x) d x
$$

so that the saddle node is exactly at $\alpha=0$. Note that at the saddle node bifurcation,

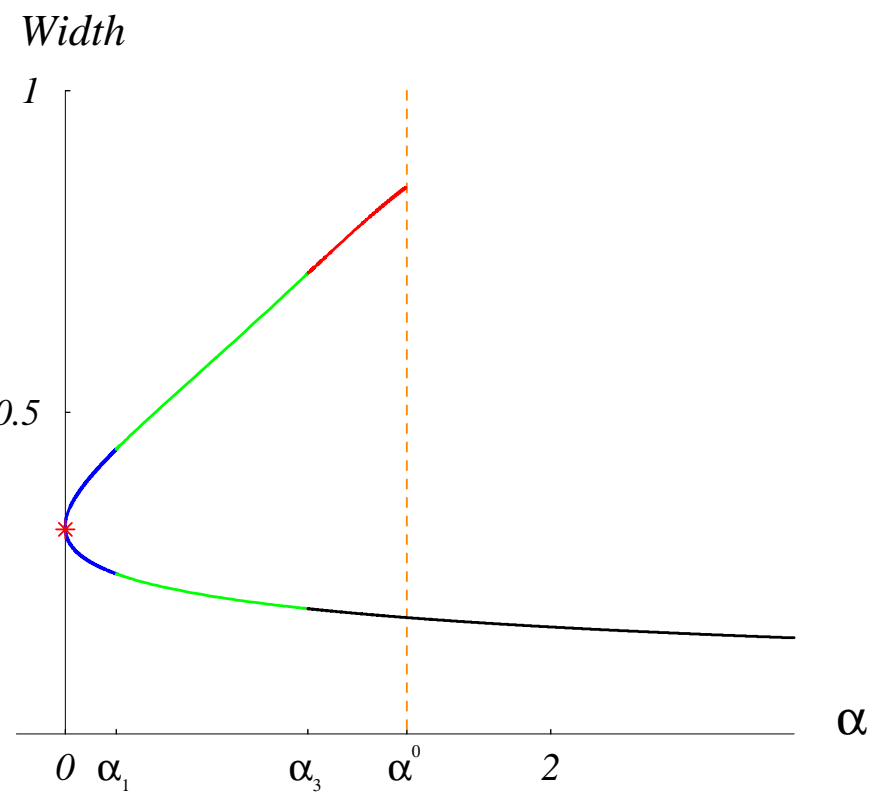

FIG. 4.1. Width of single-pulse $\mathbf{l}$ (upper branch) and $\mathbf{s}$ (lower branch) for $a=2.6$, $A=2.8$, and $u_{T}=0.400273$. For $\alpha \in\left[\alpha^{*}, \alpha^{0}\right)$, there are two single-pulses. For $\alpha \in\left[\alpha^{0}, \infty\right)$, there is only one single-pulse solution. At $\alpha=0$ there is a saddle-node bifurcation where the large single-pulse $\mathbf{1}$ and the small single-pulse $\mathbf{s}$ arise. At $\alpha^{0}$, the large single-pulse $\mathbf{1}$ blows up.

the pulse arises with nonzero height and width.

We can now track the location of the saddle node and the maximum firing rate of the pulse at the saddle node in parameter space. We reduce the four dimensional parameter space by projecting to the space $\left(\alpha, a / A, u_{T}\right)$. The saddle-node location can be found by setting the threshold $u_{T}$ to the value of the first local maximum of $\Phi(x)$. This gives an upper bound for allowable thresholds of the gain function to support a pulse solution. As long as $u_{T}$ is below this upper bound and positive, a single-pulse can exist.

We first set $A=1.5$ and vary the ratio $a / A$ and $\alpha$ to identify the saddle node threshold $u_{T}$. We then calculate the maximum firing rate $f_{\max }$ of the single-pulse 


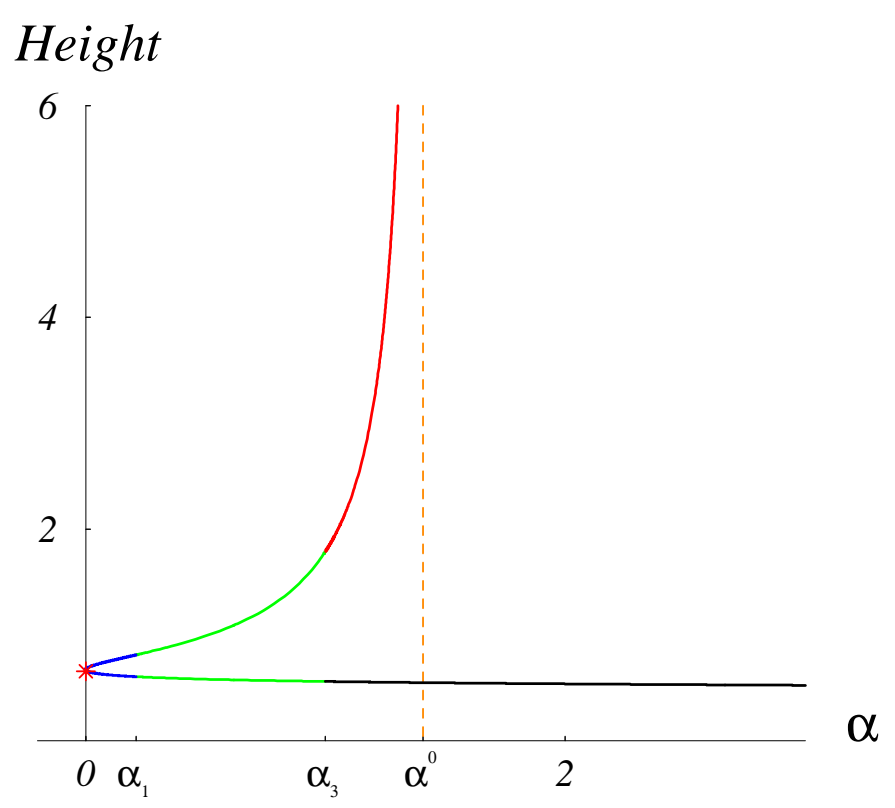

FIG. 4.2. Height of single-pulse $\mathbf{l}$ (upper branch) and $\mathbf{s}$ (lower branch) for the same conditions as Fig. 4.1 .

solution at this threshold which creates a two dimensional surface in the space of $\left(a / A, \alpha, u_{T}\right)$. We increment $A$ in steps of 1 and create a set of surfaces. The surface plots of $u_{T}$ and $f_{\max }$ vs $a / A$ and $\alpha$ are shown in Figs. 4.3 and 4.4 Single-pulse solutions exist below a given surface (with $u_{T}>0$ ). Depending on the parameters, solutions could include one single-pulse $\mathbf{s}$, a coexistence of single-pulses $\mathbf{s}$ and $\mathbf{l}$ (or a dimple-pulse $\mathbf{d}$ but in a smaller global range), or coexistence of more than two pulses.

When excitation dominates inhibition (i.e. $a / A$ is small) the single-pulse solution can blow up as mentioned in Sec. 3.5.3. We note that the crucial parameter for maintaining low firing rates is for inhibition to dominate excitation (i.e. $a / A$ to be large). Even for the balanced case of $a=A$, for gain slope $\alpha$ beyond unity, the firing rate rises dramatically. This is in correspondence with observations of numerical simulations 69, 70.

5. Construction of double-pulse solutions. The neural network equation (1.1) can also support double-pulse or even multiple-pulse solutions [32, 42. Doublepulses are solutions that have two disjoint open and finite intervals for which the synaptic input $u(x)$ is above threshold.

Definition 5.1. Double-pulse solution: A solution $u(x)$ of 2.4) is called a double-pulse or a 2-pulse if there are $x_{1}>0$ and $x_{2}>0$ such that

$$
u(x) \begin{cases}>u_{T} & \text { if } x \in\left(x_{1}, x_{2}\right) \cup\left(-x_{2},-x_{1}\right), x_{1,2}>0 \\ =u_{T} & \text { if } x=-x_{2},-x_{1}, x_{1}, x_{2} \\ <u_{T} & \text { otherwise }\end{cases}
$$

with

$$
\left(u, u^{\prime}, u^{\prime \prime}, u^{\prime \prime \prime}\right) \rightarrow(0,0,0,0)
$$

exponentially fast as $x \rightarrow \pm \infty . u$ and $u^{\prime}$ are bounded and continuous on $R . u^{\prime \prime}, u^{\prime \prime \prime}$ and $u^{\prime \prime \prime \prime}$ are continuous everywhere for $x \in \Re$ except $x= \pm x_{1,2}$ and bounded everywhere 


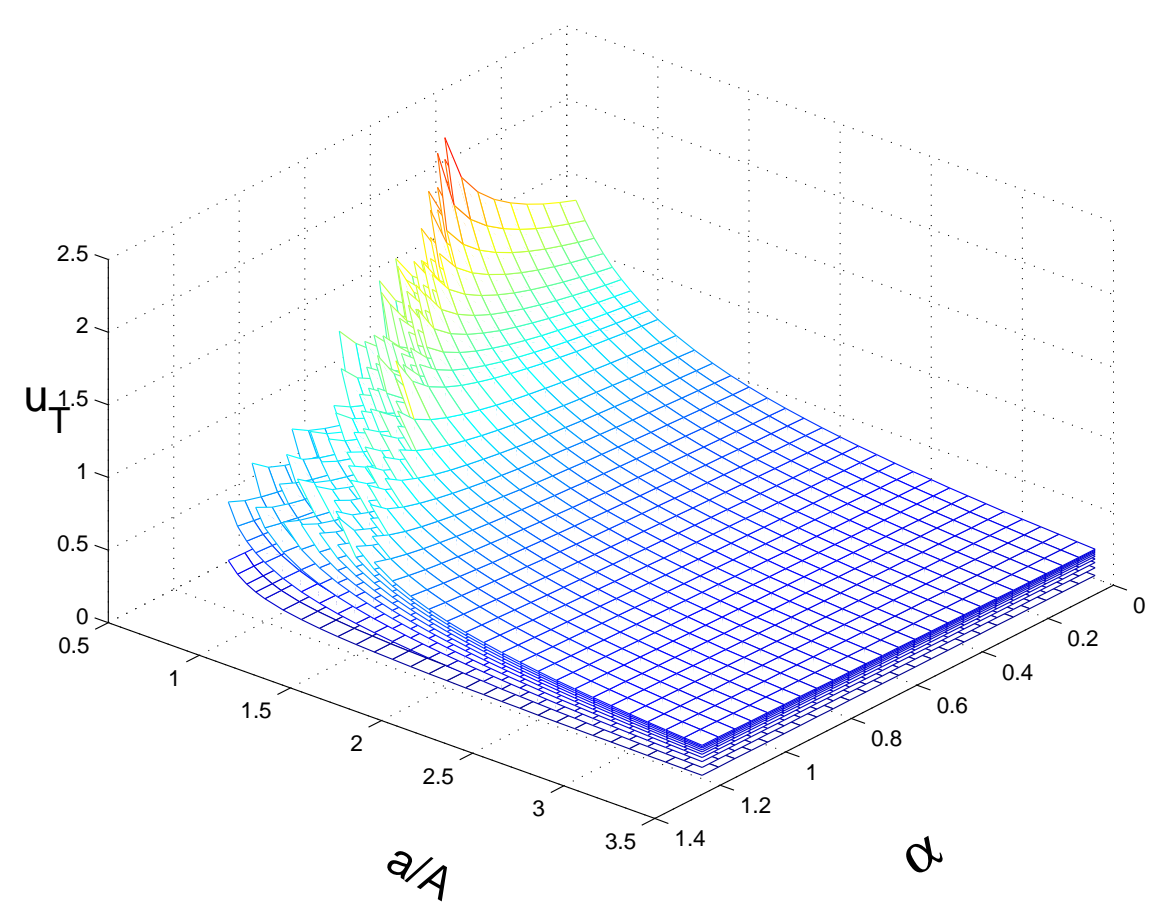

FIG. 4.3. Surface plot of saddle node point in parameter space of $\left(a / A, \alpha, u_{T}\right)$. The separate leaves correspond to values of A ranging from [*] to [*].

on $R . u(x)$ is symmetric with $u^{\prime \prime}(0)>0 ; u(0)$ is the minimum between $-x_{1}$ and $x_{1}$ (Fig. 5.2).

The approach to find and construct double-pulse solutions is similar to that for single-pulses. The connection function is (2.1) and the gain function is (2.2). Laing and Troy 42 found that double pulses can exist for the Amari case $(\alpha=0)$. However, for the exponential connection function (2.1), the double pulses are unstable. Coombes et al. [15] found that double and higher number multiple-pulse solutions could exist in a network with a saturating sigmoidal gain function. As in the single-pulse case, a fourth order ODE on $x \in(-\infty, \infty)$ for double-pulses can be derived:

$$
\begin{aligned}
& u^{\prime \prime \prime \prime}-\left(a^{2}+1\right) u^{\prime \prime}+a^{2} u= \\
& 2 a(A-a) f[u(x)]+2(a A-1)\left\{f\left[u\left(x_{2}\right)\right] \Delta_{2}^{\prime}(x)-f\left[u\left(x_{1}\right)\right] \Delta_{1}^{\prime}(x)\right\}- \\
& 2(a A-1)\left\{f^{\prime}\left[u\left(x_{1}\right)\right] u^{\prime}\left(x_{1}\right) \Delta_{1}(x)+f^{\prime}\left[u\left(x_{1}\right)\right] u^{\prime}\left(x_{1}\right) \Delta_{2}(x)\right\}- \\
& 2(a A-1) \frac{d^{2} f[u(x)]}{d x^{2}}
\end{aligned}
$$

where

$$
\begin{aligned}
& \Delta_{1}(x)=\delta\left(x-x_{1}\right)+\delta\left(x+x_{1}\right) \\
& \Delta_{2}(x)=\delta\left(x-x_{2}\right)+\delta\left(x+x_{2}\right) \\
& \Delta_{1}^{\prime}(x)=\delta^{\prime}\left(x-x_{1}\right)-\delta^{\prime}\left(x+x_{1}\right) \\
& \Delta_{2}^{\prime}(x)=\delta^{\prime}\left(x-x_{2}\right)-\delta^{\prime}\left(x+x_{2}\right)
\end{aligned}
$$

Double-pulses can be constructed using ODE (5.1) and the following set of matching 


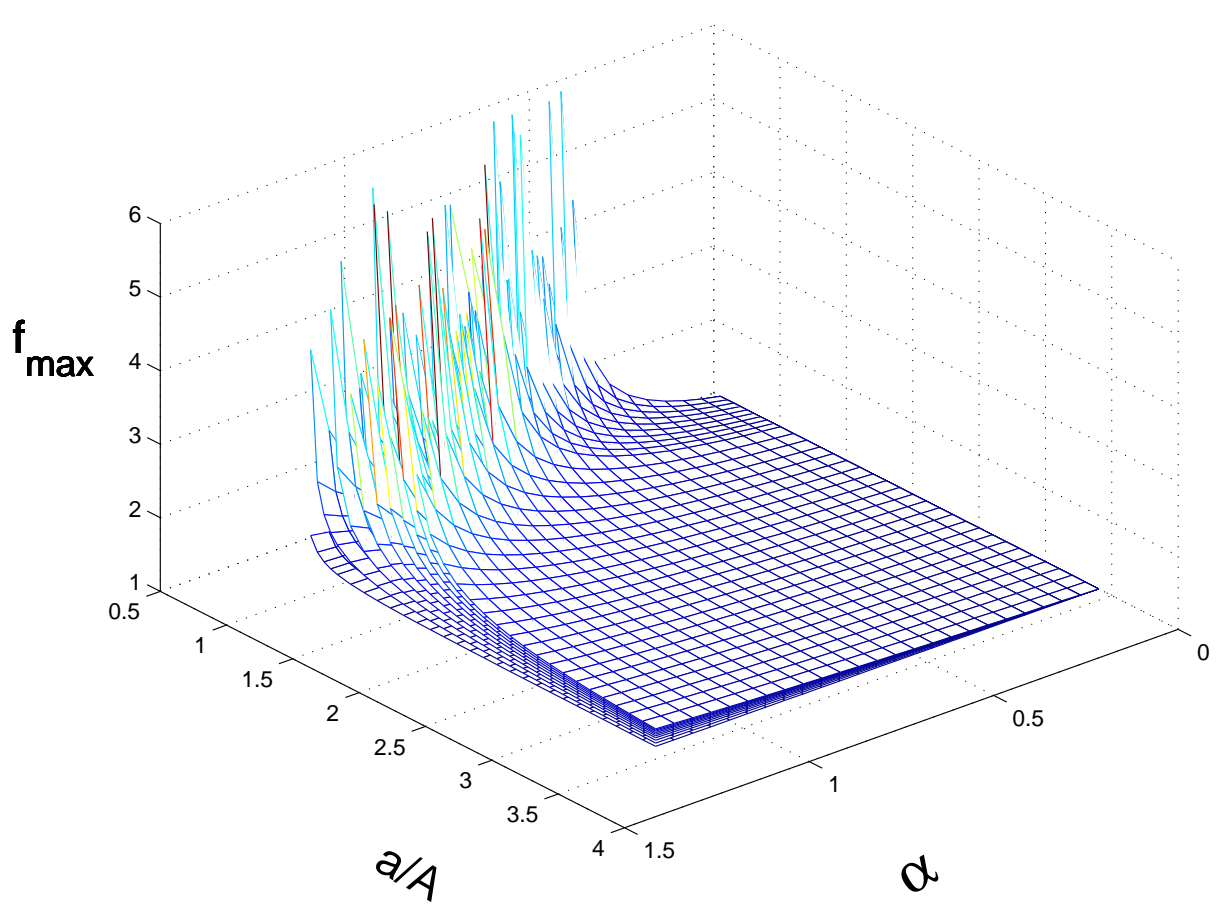

FIG. 4.4. Maximum point of firing rate of the single-pulse at the saddle node for the conditions as Fig. 4.3

conditions (5.2)-(5.11) at both $x_{1}$ and $x_{2}$ :

$$
\begin{aligned}
u_{I}\left(x_{1}\right) & =u_{T} \\
u_{I I}\left(x_{1}\right) & =u_{T} \\
u_{I I}\left(x_{2}\right) & =u_{T} \\
u_{I I I}\left(x_{2}\right) & =u_{T} \\
u_{I}^{\prime}\left(x_{1}\right) & =u_{I I}^{\prime}\left(x_{1}\right) \\
u_{I}^{\prime \prime}\left(x_{1}\right) & =u_{I I}^{\prime \prime}\left(x_{1}\right)+2(a A-1) f\left(u\left(x_{1}\right)\right) \\
u_{I}^{\prime \prime \prime}\left(x_{1}\right) & =u_{I I}^{\prime \prime \prime}\left(x_{1}\right)+2(a A-1) f^{\prime}\left(u\left(x_{1}\right)\right) u^{\prime}\left(x_{1}\right) \\
u_{I I}^{\prime}\left(x_{2}\right) & =u_{I I I}^{\prime}\left(x_{2}\right) \\
u_{I I}^{\prime \prime}\left(x_{2}\right) & =u_{I I I}^{\prime \prime}\left(x_{2}\right)-2(a A-1) f\left(u\left(x_{2}\right)\right) \\
u_{I I}^{\prime \prime \prime}\left(x_{2}\right) & =u_{I I I}^{\prime \prime \prime}\left(x_{2}\right)-2(a A-1) f^{\prime}\left(u\left(x_{2}\right)\right) u^{\prime}\left(x_{2}\right)
\end{aligned}
$$

In the Amari case $(\alpha=0)$, for the same set of values of $a, A, u_{T}$ we find that there are two coexisting double-pulse solutions. The existence conditions are

$$
\begin{aligned}
& f_{1}\left(x_{1}, x_{2}\right)=u\left(x_{1}\right)=\int_{-x_{2}}^{-x_{1}} w\left(x_{1}-y\right) d y+\int_{x_{1}}^{x_{2}} w\left(x_{1}-y\right) d y=u_{T} \\
& f_{2}\left(x_{1}, x_{2}\right)=u\left(x_{2}\right)=\int_{-x_{2}}^{-x_{1}} w\left(x_{2}-y\right) d y+\int_{x_{1}}^{x_{2}} w\left(x_{2}-y\right) d y=u_{T}
\end{aligned}
$$

Both $x_{3}=f_{1}\left(x_{1}, x_{2}\right)$ and $x_{3}=f_{2}\left(x_{1}, x_{2}\right)$ form two dimensional surfaces in the three dimensional space $\left(x_{1}, x_{2}, x_{3}\right)$. The two surfaces intersect in a convex up space curve. 
The intersection of this space curve with the plane $x_{3}=u_{T}$ are widths of candidate double pulse solutions. Since the surfaces do not oscillate for the Amari case there are only two intersection points. These two points give two double pulse solutions which are shown in Fig. 5.1 For the general $\alpha>0$ case we, have found two coexisting double-pulses as shown in Fig 5.2 The coexistence of more than two double-pulses for $\alpha>0$ remains an open problem.

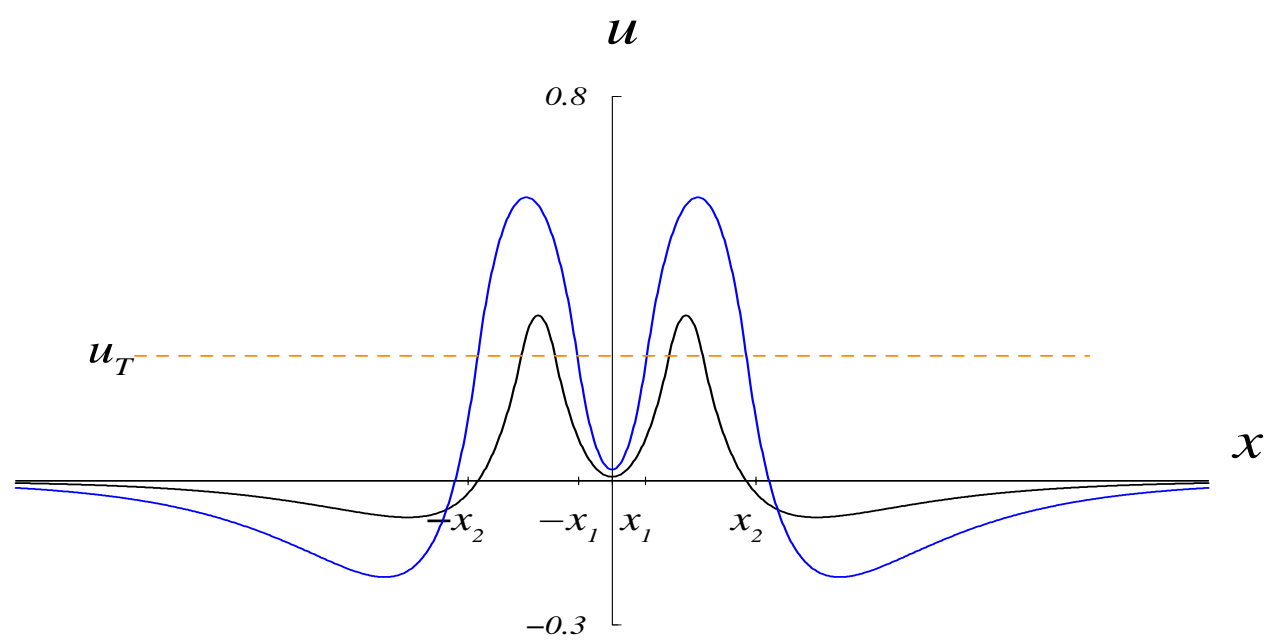

Fig. 5.1. Double-pulse for Amari case in which $\alpha=0 . \quad A=2.8, a=2.6, \alpha=0$, $u_{T}=0.26$. For the large double-pulse(blue): $x_{1}=0.279525, x_{2}=1.20521$. For the small double-pulse (black): $x_{1}=0.49626, x_{2}=0.766206$.

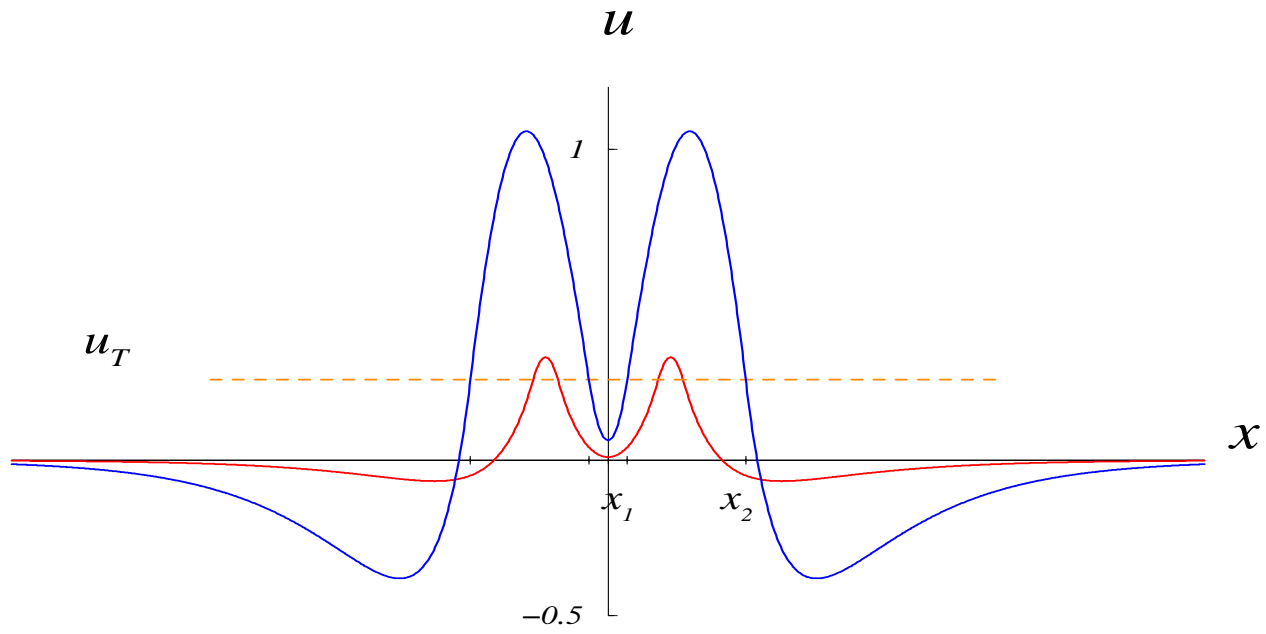

FIG. 5.2. Two coexisting double-pulses. $A=2.8, a=2.6, \alpha=0.98, u_{T}=0.26$. For the large double-pulse (blue): $x_{1}=0.19266, x_{2}=1.38376$. For the small double-pulse (red): $x_{1}=0.50582, x_{2}=0.752788$.

In the accompanying paper [33] we confirm the Laing and Troy finding [42] that the double pulses in the Amari case are unstable. 
6. Discussion. In this paper, we consider a population neural network model of the form (1.1) with a nonsaturating gain function of the form (1.2). We show the existence of stationary solutions that satisfy the equilibrium equation (2.4) by explicitly constructing single-pulse solutions for a specific synaptic connection function (2.1). The strategy was to convert the integral equation (2.4) into a fourth order ordinary differential equation. A proof for the existence of a single-pulse of (2.4) then becomes a proof for the existence of a homoclinic orbit of the ODE. Since the ODE has discontinuities across the threshold points, the ODE on the real line is reduced to three different linear ODEs on three regions separated by threshold points. The matching conditions for the solutions of the ODEs across the threshold points must satisfy a system of five equations. From this system, we are able to construct different single-pulse solutions.

The eigenvalue structure of the linear ODEs is important for determining how many pulses exist. For real $\omega_{1}$ and $\omega_{2}$, there are at most two pulses, the small single-pulse and the large one. Amari's case $(\alpha=0)$ belongs to this regime. The large single-pulse can transform to a dimple-pulse depending on the threshold value (Fig. 3.10) If the eigenvalues are complex, there could be a small single-pulse and two large pulses with different widths. Depending on the threshold, these two large pulses could be dimple-pulses (Fig. 3.17) There also exists a transition point where a dimple-pulse breaks into a double-pulse.

There are three ways that the large pulse can disappear. First, for fixed gain $\alpha$ and threshold $u_{T}$, if the excitation is too strong, i.e. ratio $A / a$ is large, the width of the large pulse becomes wider and eventually loses existence. Second, with fixed excitation, i.e., fixed $a$ and $A$, if the gain is too large, i.e., $\alpha$ is large, the large pulse increases in height and blows up at a finite value of $\alpha$. Third, with too little excitation or gain, the stable large pulse coalesces with the unstable small pulse and vanishes in a saddle node bifurcation.

The pulses are a proposed mechanism of persistent neuronal activity observed during working memory. Therefore, it is crucial to access their stability. In the accompanying paper, we show that the large pulse is stable and the small pulse is unstable. We also show that dimple-pulses can be stable. We show that singlepulses can exist for a wide variety of gain and connection functions. However, for single-pulses to exist with low firing rates we require the gain to not be too large and inhibition to dominate excitation. This suggests that the cortex could be dominated by inhibition.

Acknowledgment: We would like to thank G. Bard Ermentrout, Jonathan Rubin, Bjorn Sandstede and William Troy for illuminating discussions. This work was supported by the National Institute of Mental Health and the A.P. Sloan foundation.

\section{REFERENCES}

[1] C. D. Aliprantis. Problems in real analysis: a workbook with solutions. Academic Press, 1999.

[2] C. D. Aliprantis and Burkinshaw. Principles of real analysis. Academic Press, 1998.

[3] S. Amari. Dynamics of pattern formation in lateral-inhibition type neural fields. Biol. Cybernetics, 27:77-87, 1977.

[4] M. A. Arbib, editor. The Handbook of Brain Theory and Neural Networks. MIT Press, 1995.

[5] K. E. Atkinson. Numerical solution of integral equations of the second kind. Cambridge University Press, 1997.

[6] A. Baddeley. Working Memory. Oxford University Press, 1986. 
[7] R. Ben-Yishai, R. Lev Bar-Or, and H. Sompolinsky. Theory of orientation tuning in visual cortex. Proc. Natl. Acad. Sci. USA, 92:3844-3848, 1995.

[8] C. M. Bender and S. A. Orszag. Advanced mathematical methods for scientists and engineers I: Asyptotic methods and perturbation theory. Springer, 1999.

[9] W. E. Boyce and R. C. DiPrima. Introduction to differential equations. John Wiley and Sons, 1970.

[10] M Camperi and X.J. Wang. A model of visuospatial working memory in prefrontal cortex: recurrent network and cellular bistability. J. Comp. Neurosci, 5:383-405, 1998.

[11] A. R. Champneys and J. P. McKenna. On solitary waves of a piece-wise linear suspended beam model. Nonlinearity, 10:1763-1782, 1997.

[12] C. L. Cobly, J.-R. Duhamel, and M. E. Goldberg. Oculocentric spatial representation in parietal cortex. Cerebral Cortex, 5:470-481, 1995.

[13] A Compte, C. Constantinidis, J Tegnr, S. Raghavachari, M.V. Chafee, P.S. Goldman-Rakic, and X-J Wang. Temporally irregular mnemonic persistent activity in prefrontal neurons of monkeys during a delayed response task. J. Neurophysiol., 9:3441-3454, 2003.

[14] B. W. Connors. Intrinsic neuronal physiology and the functions, dysfunctions and development of neocortex. Progress in Brain Research, 102:195-203, 1994.

[15] S. Coombes, G. J. Lord, and M. R. Owen. Waves and bumps in neuronal networks with axo-dendritic synaptic interactions. Physica D, 178:219-241, 2002.

[16] L. M. Delves and J. L. Mohamed. Computational methods for integral equations. Cambridge University Press, 1988.

[17] D. G. Duffy. Green's functions with applications. Chapman and Hall/CRC, 2001.

[18] S. A. Ellias and S. Grossberg. Pattern formation, contrast control, and oscillations in the short-term memory of shunting on-center off-surround networks. Biol. Cybern., 20:69-98, 1975.

[19] G. B. Ermentrout. Xppaut, simulation software tool.

[20] G. B. Ermentrout. Reduction of conductance-based models with slow synapses to neural nets. J. Math. Biology, 6:679-695, 1994.

[21] G. B. Ermentrout. Neural networks as spatio-temporal pattern-forming systems. Rep. Prog. Phys., 61:353-430, 1998.

[22] G. B Ermentrout. Simulating, Analyzing, and Animating Dynamical Systems: A Guide to XPPAUT for Researchers and Students. SIAM, 2002.

[23] G. B. Folland. Fourier analysis and its applications. Wadsworth and Brooks/Cole Advanced Books and Software, 1992.

[24] S. Funahashi, Bruce G. J., and P.R. Goldman-Rakic. Mnemonic coding of visual space in the monkey's dorsolateral prefrontal cortex. J. Neurophys., 61:331-349, 1989.

[25] J. Fuster and G. Alexander. Neuron activity related to short-term memory. Science, 173:652654, 1971.

[26] J. M Fuster. Prefrontal cortex: anatomy, physiology, and neuropsychology of the frontal lobe. Lippincott-Raven Publishers, 1997.

[27] F. Garvan. The Maple Book. Chapman and Hall, 2001.

[28] P. S. Goldman-Rakic. Cellular basis of working memory. Neuron, 14:477-485, 1995.

[29] C. D. Green. Integral equation methods. Nelson, 1969.

[30] D. H. Griffel. Applied functional analysis. Ellis Horwood, 1985.

[31] S. Grossberg and D. Levine. Some developmental and attentional biases in the contrast enhancement and short-term memory of recurrent neural networks. Journal of Theoretical Biology, 53:341-380, 1975.

[32] Y. Guo. Existence and stability of standing pulses in neural networks. PhD thesis, University of Pittsburgh, 2003.

[33] Y. Guo and C.C. Chow. Existence and stability of standing pulses in neural networks: I. stability. Preprint, 2004.

[34] B.S. Gutkin, C.R. Laing, C. L. Colby, C.C. Chow, and G. B. Ermentrout. Turing on and off with excitation: the role of spike-timing asynchrony and synchrony in sustained neural activity. J. Comp. Neurosci., 11:121-134, 2001.

[35] D. Hansel and H. Sompolinsky. Modeling Feature Selectivity in Local Cortical Circuits. In Methods in Neuronal Modeling: From Synapse to Networks. Koch C and Segev I. Eds, 
(MIT Press, Cambridge, MA, Chapter 13, second edition, 1998.

[36] E Haskell and P. C. Bressloff. On the formation of persistent states in neuronal networks models of feature selectivity. J. Integ. Neurosci., 2:103-123, 2003.

[37] M. Kang, K.and Shelley and H. Sompolinsky. Mexican hats and pinwheels in visual cortex. PNAS, 100:2848-2853, 2003.

[38] T. Kato. Perturbation theory for linear operators. Springer, 1995.

[39] K Kishimoto and S. Amari. Existence and stability of local excitations in homogeneous neural fields. J. Math. Biology, 7:303-318, 1979.

[40] Y. A. Kuznetsov. Elements of applied bifurcation theory. Springer, 1998.

[41] C. R. Laing and C. C. Chow. Stationary bumps in networks of spiking neurons. Neural Comp., 13:1473-1493, 2001.

[42] C. R. Laing and W. C. Troy. Two-bump solutions of amari type models of working memory. Physica D, pages 190-218, 2003.

[43] C. R. Laing, w. C. Troy, B Gutkin, and G. B Ermentrout. Multiple bumps in a neuronal model of working memory. SIAM J. of Applied Math., 63, no.1:62-97, 2002.

[44] C. R. Laing and William C. Troy. Pde methods for nonlocal models. SIAM Journal on Applied Dynamical Systems, 2:487-516, 2001.

[45] P. E. Latham, B.G. Richmond, P.G. Nelson, and S. Nirenberg. Intrinsic dynamics in neuronal networks. i. theory. J. Neurophysiol., 83(2):808-827, 2000.

[46] P. E. Latham, B.G. Richmond, S. Nirenberg, and P.G. Nelson. Intrinsic dynamics in neuronal networks. ii. experiment. J. Neurophysiol., 83(2):828-835, 2000.

[47] D.A. McCormick, Y.-S. Shu, A Hasenstaub, M. Sanchez-Vives, M. Badoual, , and T. Bal. Cellular and network mechanisms of rhythmic, recurrent activity in the cerebral cortex. Cerebral Cortex, 13:1219-1231, 2003.

[48] E. K. Miller, C. A. Erickson, and R. Desimone. Neural mechanisms of visual working memory in prefrontal cortex of the macaque. J. Neurosci., 16:5154-5167, 1996.

[49] N. Morrison. Introduction to Fourier analysis. Wiley-Interscience, 1994.

[50] J. D. Murray. Mathematical biology. Springer, 2002.

[51] J. G. Nicholls. From neuron to brain: a cellular molecular approach to the function of the nervous system. Sinauer Associates, 1992.

[52] Y. Nishiura and M. Mimura. Layer oscillations in reactoin-diffusion systems. SIAM J. Appl. Math., 49:481-514, 1989.

[53] E. Pärt-Enander, A. Sjöberg, Melin B., and Isaksson P. The MATLAB handbook. AddisonWesley, 1998.

[54] L. A. Peletier and W. C. Troy. Spatial patterns: higher order models in physics and mechanics. Birkhauser, 2001.

[55] E. Pelinovsky, D and V. G. Yakhno. Generation of collective-activity structures in a homogeneous neuron-like medium. i. bifurcation analysis of static structures,. Bifurcation Chaos Appl. Sci. Eng., 6:81-87, 89-100, 1996a,b.

[56] J. D. Pinto and Ermentrout G. B. Spatially structured activity in synaptically coupled neuronal networks:1 traveling fronts and pulses. SIAM J. Appl. Math., 62:206-225, 2001.

[57] J. D. Pinto and Ermentrout G. B. Spatially structured activity in synaptically coupled neuronal networks:2 lateral inhibition and standing pulses. SIAM J. Appl. Math., 62:226-243, 2001.

[58] A. D. Polianin and A. V. Manzhirov. Handbook of integral equations. CRC Press, 1998.

[59] D. L. Powers. Boundary value problems. Harcourt Academic Press, 1999.

[60] M. Rahman. Complex variables and transform calculus. Computational Mechanics Publications, 1997.

[61] A. Renart, N. Brunel, and Wang X-J. Mean-field theory of recurrent cortical networks: from irregularly spiking neurons to working memory. in Computational Neuroscience: A Comprehensive Approach. J. Feng Ed., CRC Press, Boca Raton, 2003.

[62] J. E. Rubin, D. Terman, and C. C. Chow. Localized bumps of activity sustained by inhibition in a two-layer thalamic network. J Comp Neurosci, 10:313-331, 2001.

[63] J. E Rubin and W. C Troy. Sustained spatial patterns of activity in neuronal populations with or without lateral inhibition. SIAM J. Appl. Math., 2004.

[64] W. Rudin. Principles of mathematical analysis. McGraw-Hill, 1976.

[65] E. Salinas and L. F. Abbott. A model of multiplicative neural responses in parietal cortex. 
Proc Natl Acad Sci USA, 93:11956-11961, 1996.

[66] M.V. Sanchez-Vives and D.A. McCormick. Cellular and network mechanisms of rhythmic, recurrent activity in the cerebral cortex. Nature Neuroscience, 3:1027-1034, 2000.

[67] S. H. Seung. How the brain keeps the eyes still. Proc Natl Acad Sci USA, 93:13339-44, 1996.

[68] S. H. Strogatz. Nonlinear dynamics and chaos. Perseus Books, 1994.

[69] X-J Wang. Synaptic basis of cortical persistent activity: the importance of nmda receptors to working memory. J. Neuroscience, 19(21):9587-9603, 1999.

[70] X-J Wang. Synaptic reverberation underlying mnemonic persistent activity. Trends in Neurosci, 24:455-463, 2001.

[71] S. Wiggins. Introduction to applied nonlinear dynamical systems and chaos. Springer, 1990.

[72] H. R. Wilson and J. D. Cowan. Excitatory and inhibitory interactions in localized populations of model neurons. Biophys. J., 12:1-24, 1973.

[73] H. R. Wilson and J. D. Cowan. A mathematical theory of the functional dynamics of cortical and thalamic nervous tissue. Kybernetic, 13:55-80, 1973.

[74] S. Wolfram. The Mathematica Book. Cambridge University Press, 4th Edition, 1999.

[75] E. Zeidler. Nonlinear functional analysis and its applications I: fixed-point theorems. Springer, 1986. 